\title{
Beiträge zur Kenntnis der Lias-Volen Südamerikas und der Stammesgeschichte der Gattung Vola
}

\author{
Von Dr. E. Jaworski in Bonn
}

\section{Einleitung}

Die Gattung Vola KLEIN = Janira ScHUM. $=$ Neithea DrouET $=$ Pecten s. str. FISCHER (22 S. 946) u. (16 S. 9), charakterisiert durch konvexe R. und konkave bis flache Lk. Kl., mit stark verkümmertem bis fehlendem Byssusausschnitt (31 S. 111), tritt zum ersten Male im Lias von Südamerika und zwar nur von Südamerika auf, wo sie uns sofort sehr individuenreich und, wie es neuerdings scheint (32), auch recht artenreich begegnet, mit z. T. großen und mannigfach skulpturierten Formen. Ihr dortiges Vorkommen gab sogar lange Zeit Veranlassung, entsprechend unseren europäischen Erfahrungen, die durch sie charakterisierten Schichten nicht als Jura, sondern als Kreide aufzufassen.

Ein besonderes Interesse verdienen diese Lias-Janiren durch die an sie sich knüpfenden phylogenetischen Untersuchungen, vor allem von PHIIIPPI (31). (Vergl. S. 311-12 dieser Arbeit.) Ein von mir bearbeitetes reiches $Z$ weischalermaterial aus dem südamerikanischen Lias (26) gab mir Veranlassung, mich näher mit diesen Formen zu befassen, und es zeigte sich hierbei, daß deren bisherige Darstellung in der Literatur ${ }^{1}$ ) textlich wie bildlich vielfach unrichtig und unvollständig ist; auch die Kombination der einzelnen Darstellungen gibt kein klares Bild; viele Einzelheiten, die mein Material erkennen läßt, sind neu. Aus diesem Grunde erschien mir der z. T. ideal schöne Erhaltungszustand dieses

1) Literatur vergl. in den Literaturangaben des I. Teiles.

Zeitschr. f. Palaeontologie. Bd. I. 
Materials als eine willkommene Gelegenheit zur Ausfüllung jener Lücke. Der erste Teil der vorliegenden Arbeit bringt eine ausführliche Beschreibung und Neuabbildung der Formen. Im Teil II habe ich nochmals die phylogenetischen Beziehungen der Lias-Volen zu den jüngeren Vertretern dieser Gattung untersucht und bin auf Grund meiner Beobachtungen zu Resultaten gekommen, die denen PHILIPPI's, der übrigens die Lias-Volen sehr kurz erledigt, z. T. direkt entgegengesetzt sind. Es scheint vor allem erweisbar, daß a) aus Vola Pectiniden entstanden sind, nicht umgekehrt, b) die Tertiär-Volen Nachkommen der Liasformen sind, also keine selbständigen Iterativbildungen, c) die Kreide-Volen z. T. Nachkommen der Lias-Volen, zum größten Teil aber anderen noch unbekannten Ursprungs sind.

Das Material stammt aus den verschiedensten Teilen Südamerikas und wurde mir von Herrn Geh.-Rat STELNMANN zur Bearbeitung überlassen, auf dessen Anregung ich hauptsächlich nochmals die Phylogenie von Vola nachgeprüft habe. Es ist mir eine angenehme Dankespflicht, meinem hochverehrten Lehrer an dieser Stelle nochmals für das Interesse und seine vielfachen Ratschläge, deren ich mich bei der Anfertigung dieser Arbeit zu erfreuen hatte, meinen herzlichsten Dank auszusprechen.

Bezüglich stratigraphischer und geologischer Einzelheiten, sowie der Lage der Fundpunkte verweise ich auf meine ausführliche Arbeit über südamerikanischen Jura (26).

\section{Teil. Beschreibung des Materials}

\section{A. Lias-Volen}

Über die Abgrenzung und Artberechtigung der verschiedenen Spezies sind die Ansichten sehr geteilt. Von den zahlreichen PHسIPPI'schen Arten, mit Ausnahme seines Pecten alatus BAYLE et COQU. $=P$. commutatus PसII. abgesehen, hat man bisher unterschieden:

1. Vola alata v. Buch $\left\{\begin{array}{l}\text { v. Buch (9) T. 1, Fig. 1-4. } \\ \text { BAYLE et Coqu. (2) T. 5, Fig. 1-2. } \\ \text { BuRCKHARDT (11) T. 19, Fig. 12. }\end{array}\right.$

2. "Bodenbenderi BkHr. BhHRendsen (3) T. 22, Fig. 3.

3. "Dufrenoyi D'ORB. D'ORBIGNY (18) T. 22, Fig. 5-9.

4. " aff. alata v. BucH, RorH usw. (36) T. 10, Fig. 1-2.

5. "alata (v. BucH) BAYLE et Coqu. = commutata PHIL. BAYLE et CoQU. (2) T. 5, Fig. 1-2; Pricipri (32) T. 41, Fig. 1-2. 
Allseitig anerkannt ist die Selbständigkeit der allerdings recht variablen $V$. alata v. BucH, und ebenso selbständig ist $V$. Bodenbenderi BEHR., mit welcher $V$. aff. alata $\nabla$. BUCH identisch ist (vergl. S. 285). Ob V. Dufrenoyi eine selbständige Art ist, kann ich an der Hand meines Materials ebensowenig entscheiden wie MörICKE. D'ORBIANY, PHILIPPI und BEHREDSEN nahmen das an, während $\nabla$. BUCH (8), BURMEISTER \& GIEBEL, BAYLE et Coquand sie mit $\nabla$. alata vereinigten. Sollte $V$. Dufrenoyi die typische Dreiteilung der Rippen besitzen, so würde ich sie als besondere Art ansprechen. Die PHIIPPI'sche Abbildung (32) T. 13, Fig. 3 ist keine $V$. Dufrenoyi, wie ein Vergleich mit der Abbildung von D'ORBIGNY zeigt; sie steht vielmehr der V. Domeykoi PHIr. (32), T. 13, Fig. 4 sehr nahe. V. alata v. BUCH und alata BAYLE et COQU. = commutata PHIL. werden von PHIIPPI auf Grund ihrer verschiedenen Form und GröBe und besonders der Schuppen auf den Rippen als verschiedene Arten unterschieden. BAYLE et COQUAND wie BURMEISTER \& GIEBEL geben nur feine, wellige Anwachsstreifen als Querverzierung an. Größe und Form sind keine unbedingten Unterschiede, da, wie GIEBEL schon hervorhebt, junge und etwas verdrückte Exemplare vorgelegen haben können. Andererseits weist der Autor daranf hin, daß die Schale nicht ganz symmetrisch ist, wie sie die französischen Forscher abbilden, sondern etwas schief. Da BAYLE et COQUAND nur ein junges Exemplar erwähnen, das Schuppenbildung, und diese nur in den Zwischenräumen der lk. Klappe aufweist, und da BURMEISTER \& GIEBEL bei ihrem umfangreichen Material nur "feine scharfe Anwachslinien“. beobachtet haben und mir ebenfalls keine beschuppten Exemplare vorliegen, so dürfte wohl die BucH'sche Abbildung mit den Schuppen auf den Rippen nur eine seltenere Varietät mit extrem scharfer Querverzierung darstellen, während die Formen BAYLE et COQUAND's, BURMEISTER \& GIEBEL's sowie meine mit feinen Anwachslinien den normalen Typus repräsentieren, eine Ansicht, die auch die französischen Autoren schon angedeutet haben. T. alata $\nabla$. BणCH und alata BAYLE et COQU. = commutata PHIL. sind also wohl keine besonderen Arten, wie PHWIPPI angibt, sondern die COQJAND'sche Abbildung und die GIEBEL'sche Beschreibung stellen den Normaltypus dar, die BUCH'sche Abbildung eine Varietät, die man vielleicht zweckmäßig mit dem Zusatz,aspera" bezeichnen könnte.

Bei der eigentlichen V. alata (v. BUCH) BAYLE et COQU. variiert die Rippenzahl in gewissen Grenzen (13-16) und ebenso das Verhältnis der Breite der Rippen zu der der Zwischenräume. Hiernach kann man verschiedene Varietäten unterscheiden, wie etwa var. Titan MöR. (29) S. 40-41. Dazu kommt, daß nach MöRICKE durch Verwitterung und 
Verdrückung die Form der Rippen und Zwischenräume sehr verschieden werden kann, wodurch sich wahrscheinlich die große Anzahl von Arten erklärt, die PHILIPPI unterscheidet und die sicher zum größten Teil gar keine selbständigen Arten sind.

\section{Zusammenstellung der Vola-Arten des Lias in Südamerika}

1. V. alata (v. BuCH) $=$ V. alata (v. BuCH) var. aspera JAw.

2. " " (v. Buch) BaYle et Coqu. = commutata PHIL. = Normaltypus.

3. "Dufrenoyi D'ORB. $=$ (V. alata (v. BucH) BAYLE et Coqu.).

4. "Bodenbenderi BeHR. = V. aff. alata (v. Buch) BuRckH.

Von PHWIPPI'schen Formen sind, wenn wir diejenigen ausschließen, von denen nur Fragmente oder eine Schale bekannt sind, sichere Volen:
1. V. Domeykoi $\mathrm{PH}$.
T. 13, Fig. 4.
2. „paragogus $\mathrm{PH}$. . . . . . . . . . T. 13, Fig. 5.
3. "Goliath Рн. . . . . . . . . . . T. 14, Fig. 1.
4. "anguste-costata Рн. . . . . . . . . T. 14, Fig. 2 u. T. 16, Fig. 1.
5. " dilatata Рн. . . . . . . . . . . T. 17, Fig. 3.
6. ”excavata Рн. . . . . . . . . . . T. 18, Fig. 1.
7. " curvicosta Pr. . . . . . . . . . T. 18, Fig. 3.
8. "Harnäckeri Рн. . . . . . . . . . T. 18, Fig. 4.
9. "subcarinata Рн. . . . . . . . . T. 19, Fig. 4.
10. "Stolpi Рн. . . . . . . . . . . . T. 19, Fig. 5.

Welche Formen hiervon wirklich selbständige Arten sind, läßt sich ohne Vergleichsmaterial nicht sicher feststellen. Auf Grund meines Materials kann ich nur sagen, daB V. Domeykoi PH. sicher eine selbstständige Art ist, und ebenso sicher, daß $V$. curvicosta $\mathrm{PH}$. nur eine verdrückte $V$. alata ist, was übrigens, auch für $V$. dilatata $\mathrm{PH}$. höchst wahrscheinlich ist.

\section{V. alata (V. BUCH) BATLE et COQU. Fig. 1-7}

V. alata (v. Buch) Bayle et Coqu., Bayle et Coqdand (2) T. 1, Fig. 1-2.

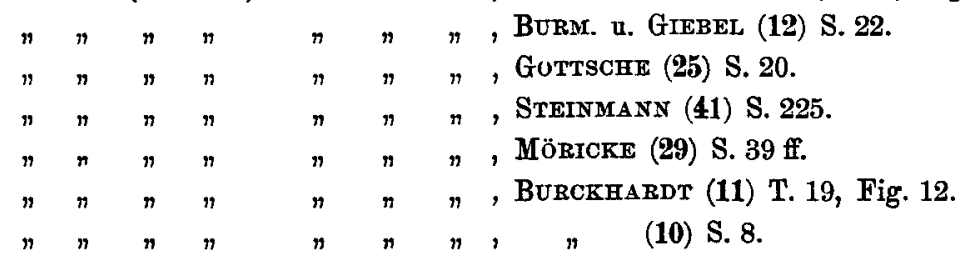


Außerdem noch zahlreiche vereinzelte Angaben; über Literatur vergl. u. a. bei GotTSCHE (25). 7 beschalte Exemplare, 6 Steinkerne, 2 Abdrücke.

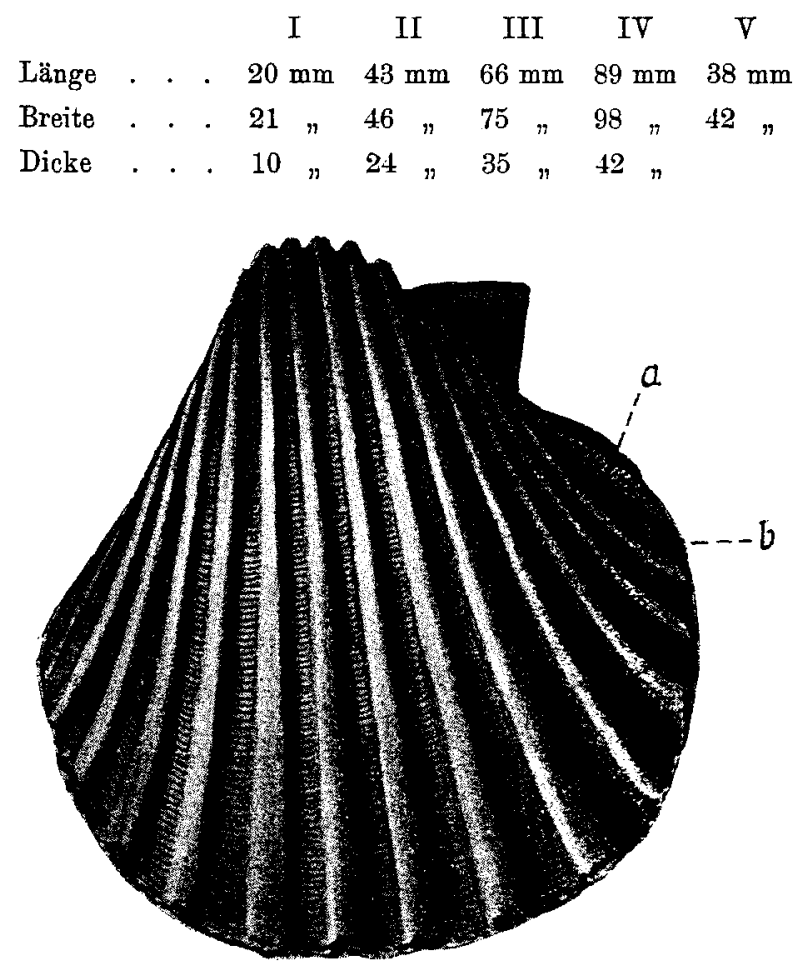

Fig. 1. Vola alata (v. Buch) BAYL et Coqu. Chunumayo, N.-Peru. Alata-Kalk des unt. Lias. Rechte Klappe. Bei a System schräg verlaufender Linien hinter der letzten Flankenrippe. Bei $b$ mehrere feine Furchen hinter der letzten Flankenrippe, dieser parallel verlaufend.

\section{Beschreibung der Schalen}

Am besten erhalten ist das abgebildete Exemplar aus Peru, das, abgesehen von der Größe, sehr gut zu den Abbildungen BAYLE et COQUAND's paßt und dessen Berippung vollkommen mit der Beschreibung von GIEBEL u. BurMeister übereinstimmt. Rechte Klappe (Fig. 1) stark konvex gewölbt. 13 scharf ausgeprägte schon in der Wirbelnähe sehr deutliche Rippen, auf dem Rücken flach konvex bis eben, schmäler wie die schwach konkaven Zwischenräume. Flankenabfall der Rippen sehr steil, fast senkrecht. Dies gilt nur für die Rippen auf der Schalenmitte; die 3 letzten Flankenrippen sind beiderseits "schiefseitig, oben kantig und ihre abfallenden Seiten buchtig"“ (12) S. 132. Auf dem Abfall der mittleren Rippen 
ist unten ein sehr schwacher Wulst zu sehen, der gewissermaßen die Rippen gegen die Zwischenräume abgrenzt, und dessen Anwesenheit den unteren Teil des steilen Rippenabfalls wie mit einer schwachen Kehle versehen erscheinen läßt (Fig. 3 bei a). Auf den mittleren Rippen nur schwach angedeutet, tritt diese Erscheinung auf den Flankenrippen deutlicher hervor. Während dieser feine Wulst auf dem kleinen Exemplar aus Peru (Fig. 2) scharf hervortritt, ist er auf den mittleren Rippen des großen Exemplars nicht mehr sicher erkennbar und auch auf den Flankenrippen undeutlicher. Hieraus folgere ich, daß diese Erscheinung nur bei jungen Exemplaren deutlich hervortritt und mit

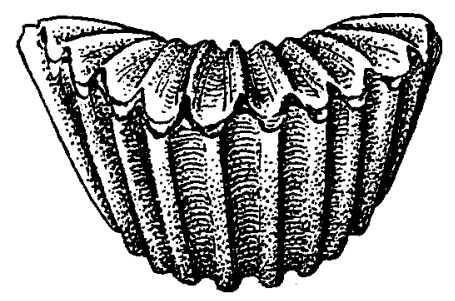

Fig. 2. Vola alata (v. BucH)

Ba Yue et Coqu. Chunumayo, Peru. Alata-Kalk des unt. Lias. Kleines Exempl. Zur Veranschaulichung der Wölbungsverhältnisse.

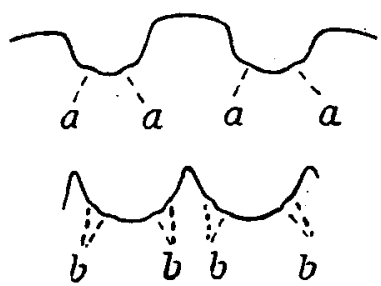

Fig. 3. Querschnitt durch die Rippen der rechten (I) und linken (II) Klappe eines gröBeren Exemplares derselben Spezies vom gleichen Fundort. $2: 1$. Bei $a$ und $b$ die feinen Wülste bezw. Kehlen anf dem Flankenabfall der Rippen.

dem Alter verschwindet. Die Zwischenräume zeigen die charakteristischen scharfen Anwachsstreifen. Zwischen der letzten schiefen Flankenrippe und dem Schalenrande bezw. Ohr findet sich ein System schief verlaufénder linienfeiner Furchen, die nur noch schwach an der hinteren Schalenseite erkennbar sind (Fig. 1 bei a). Ich kann weder bestätigen, daß - „chaque côte est divisé longitudinalement par quatre sillons symetriques, laissant dans leurs intervalles des petites côtes planes", noch daß "ses côtes sont simples", sie haben vielmehr den erwähnten schwachen rippenartigen Wulst an der Grenze gegen die Zwischenräume. Auf alle Fälle kann man die Rippen noch viel eher als einfach bezeichnen, zumal da dieser Wulst bei älteren Exemplaren zu verschwinden scheint. Schon PHIIPPI hebt hervor, daß er die vier symmetrischen Furchen von BAme et COQUAND nicht beobachten kann, und daß auch auf der COQUAND'schen Abd. nichts davon $\mathrm{zu}$ sehen ist. 
Linke Klappe (Fig. 4) ${ }^{1}$ ): Konkav, mit 13 Rippen, die genau mit der Beschreibung von BURMEISTER übereinstimmen und anf deren Flanken deutlich 2 Rippchen entlang ziehen, die gewissermaßen zur Entstehung von 2 Kehlen auf den Rippenflanken führen (Fig. 3 bei $b$ ). Zwischen den Rippen die scharfen Anwachsstreifen. Auf dem vorderen und hinteren Schalenteil sind die Rippen höher und treten viel stärker hervor, mit steilerem Seitenabfall, wie auf der Schalenmitte ${ }^{2}$ ).

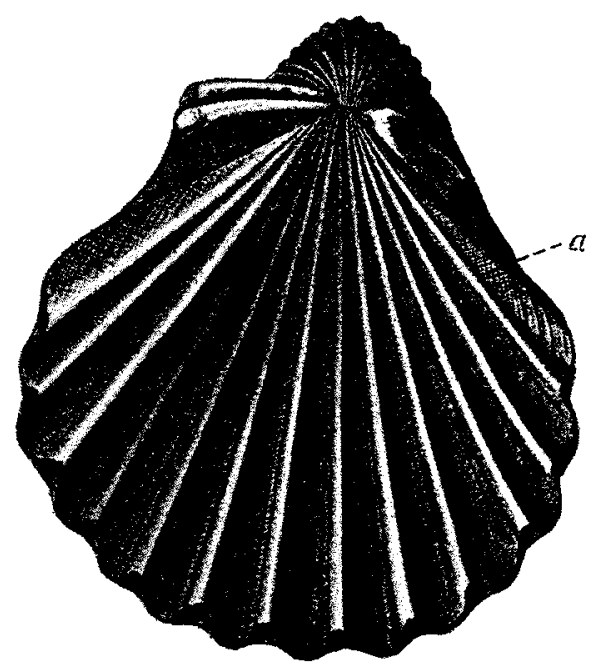

Fig. 4. Vola alata (v. Buch) BAyLe et Coqu. Chunumayo, N.-Peru.

Alata-Kalk des unt. Lias. Linke Klappe $2: 1$. Bei $a$ gitterförmiges Liniensystem zwischen der letzten Flankenrippe und dem Schalenrand.

Die Rippen sind sehr viel schmäler wie die Zwischenräume und stehen genau in den Intercostalräumen der anderen Klappe. Auf den Schalenflanken die gleichen feinen Furchen wie auf der R. Kl. Die zwischen ihnen stehenden Rippchen sind mehr oder weniger deutlich in Knötchen aufgelöst, so daß ein netzartiges Liniensystem entsteht, wie

1) Fig. 4 ist die Lk. Kl. von Fig. 2. Nachträglich ist auch die Freilegung der Lk. Kl. des großen peruanischen Exemplares (Fig. 1) gelungen. Sie stimmt mit der Abbildung des kleinen Stückes vollkommen überein.

2) Ich möchte nochmals darauf hinweisen, daß die D'ORBIGNY'sche Abbildung der Lk. Kl. vielleicht doch nichts anderes darstellen soll, wie die eben geschilderten beiden

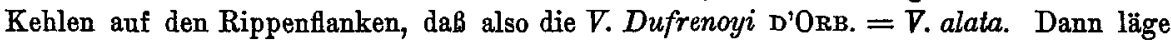
allerdings in der Zeichnung eine ganz ungeheure Übertreibung und Schematisierung der wirklichen Verhältnisse vor. 
es die Zeichnung bei a schematisiert wiedergibt. Ein ganz kleines Exemplar aus Chile stimmt bereits vollkommen mit dem beschriebenen überein, nur ist auf der Lk. Kl. die Wulstbildung auf den Flanken der Hauptrippen noch sehr undeutlich.

Ohren der beiden Klappen ohne feinere Verzierungen, nur mit Anwachsstreifen. Bei einem andern Stück befinden sich auf der R. Kl. hinter der letzten Flankenrippe mehrere rippenartige, durch feine Furchen getrennte Linien (Fig. 1 bei $b$ ), die den Flankenrippen parallel verlaufen. Dem Umriß nach stimmt gerade dieses Stück gut mit der D'ORBIGNY'schen Dufrenoyi-Abd. überein, doch ist von der typischen Dreiteilung der Rippen nichts zu sehen. In Wirbelnähe ist auf einer Rippe eine Medianfurche erkennbar, die sich in einiger Entfernung vom Wirbel verliert; sie ist schon von BURMEISTER erwähnt und als Abwitterungserscheinung gedeutet. Sehr ausgesprochen zeigt sich diese Erscheinung an den Rippen eines kleinen Exemplares von Ayasch (Peru).

Ein chilenisches Exemplar nimmt eine eigenartige Zwischenstellung zwischen $V$. alata und $V$. Domeykoi ein. Es kann mit demselben Recht zu der einen oder anderen Art gestellt werden. Ihre Form ist der PHWIPPIschen Abbildung (32) von $V$. Domeykoi genähert. Die Rippen der R. Kl. sind viel schmäler wie die Zwischenräume, fallen sanft $\mathbf{z u}$ diesen $a b$, zeigen also nicht den steilen Seitenabfall. Auf dem Rücken noch etwas flach, eben, wenn auch lange nicht in dem Maße wie bei der typischen $\nabla$. alata, geben sie aber auch noch nicht im Querschnitt das gleichmäßige wellenförmige Bild wie $\nabla$. Domeykoi. Sie sind ein Mittelding zwischen beiden. In der Wirbelgegend ist der Seitenabfall der Rippen steiler, mit Annäherung an den unteren Schalenrand wird er sanfter und flacher, und die Rippen sind auf dem Rücken mehr regelmäßig gerundet. Von den 12 Rippen der Lk. Kl. sind die äußersten Flankenrippen ziemlich scharfkantig, wie bei der jungen $V$. alata, während die mittleren etwas breiter und auf dem Rücken abgeflacht sind, besonders in der Nähe des unteren Schalenrandes. Die Schalenflanken zeigen auch hier beiderseitig das erwähnte Liniensystem von dem Aussehen eines unregelmäBigen Gitterwerkes, wie es auf der BAYLE et COQUANDschen Abbildung schon deutlich zum Ausdruck gebracht ist. Das vordere Ohr ist von mäßiger Größe, es sind nur Anwachsstreifen vorhanden. Die Innenansicht einer anderen, kleinen, Lk. Kl. zeigt eine deutliche Radialberippung der subäqualen Ohren, die von feinen Linien durchquert wird. 


\section{Beschreibung der Steinkerne Fig. 5-7}

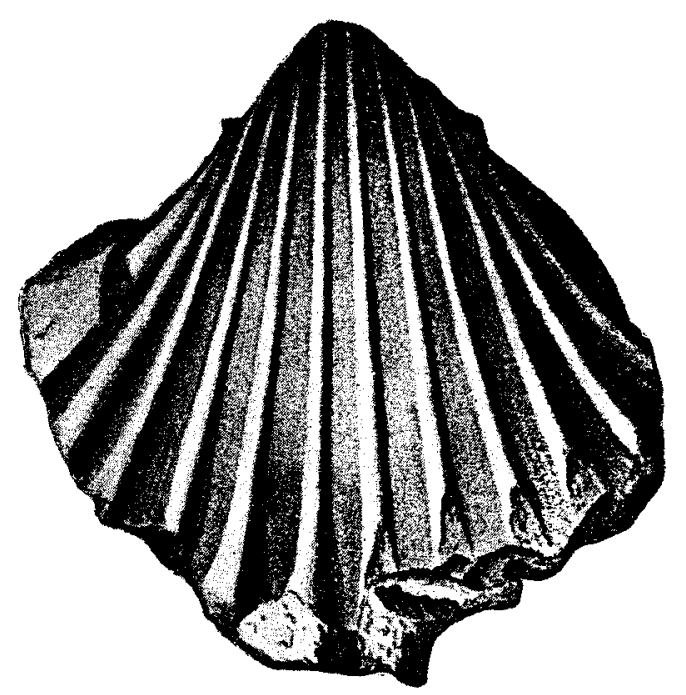

Fig. 5. Vola alaia (จ. Buch) Bayue et Coqu.

Arroyo Blanco, Rio Atuel. Unt. Atueisandstein des mittl. Lias. Steinkern der rechten Klappe.

Die Steinkerne sind meist nur schlecht und als Bruchstücke erhalten, nur ein fast vollkommener Ausguß der R. Kl. liegt vor. Zweifelsohne gehören alle die Kerne in die nächste Nähe der V. alata, ob sie aber in dem einzelnen Falle gerade von $V$. alata (v. BUCH) BAYLE et CoQU. stammen oder vielleicht von einer nahe verwandten Art, ist bei dem nahezu vollkommenen Mangel an Steinkern-Beschreibungen und -Abbildungen in dem einzelnen Falle nicht mit absoluter Sicherheit zu bestimmen. Ganz allgemein läßt sich sagen, da $B$ anf dem Steinkern die Unterschiede zwischen Rippen und Zwischenräumen nicht annähernd so scharf hervortreten wie auf der Schale; es erscheint alles viel mehr ausgeglichen. Rippen und Zwischenräume sind meistens stark konvex und zeigen auf den Flanken die gleiche Verschiedenheit gegenüber den mittleren Rippen, wie auf der Schale. Am Schalenunterrand sind die Rippen kolbenförmig verdickt und verbreitert. Das Verhältnis der Breite der Rippen und Zwischenräume zueinander schwankt etwas. An einem Steinkern sind auf den Rippen sekundäre Furchen angedentet. In der breiten Kehle, die sich dort findet, wo das Ohr an die Schale stöBt, in beiden Klappen deutliche Querrippen (Fig. 7 bei $x$ ). 
Ich habe von den Steinkernen eine Anzahl Abdrücke hergestellt, die das Bild des Schaleninnern der R. Kl. zeigen (Fig. 6). Breite Rippen, in der Mitte etwas konkav eingesenkt, mit einem außerordentlich scharfen Rand gegen die Rippenflanken abgesetzt. In der Wirbelgegend tritt dies bisweilen weniger deutlich hervor als in der Nähe des Unterrandes. Die Kante zwischen Rückenteil und Flanke der Rippen kann sogar die Form eines leichten Wulstes bekommen. Die Zwischenräume sind konkav. Am Unterrand verbreitern und vertiefen sich die Zwischenräume plötzlich stark, während die Rippen sich dementsprechend verengen. Beide sind vollkommen glatt. In Wirbelnähe sind die Rippen breiter als die Zwischenräume, mit Annäherung an den Unterrand nimmt die Breite im Verhältnis zu den Zwischenräumen ab. Die

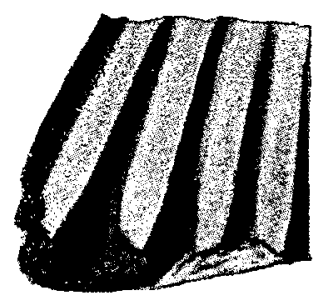

Fig. 6. Vola alata (v. Bucr) Bayle et Coqd. Arroyo Blanco, Rio Atuel, Argentinien. Unt. Atuelsandstein des mittl. Lias. Künstlicher Abdrack von einem Steinkern der rechter Klappe, der die Beschaffenheit des Schaleninnern in der Nähe des unteren Schalenrandes zeigt.

Innenansicht einer Lk. Kl. zeigt als wesentlichen Gegensatz zu den scharfen dreieckigen Rippen der Außenseite flache breite Rippen mit einem ebenen bis schwach konkaven Rücken und ebensolche Zwischenräume. Nur die zwei äußersten Flankenrippen auf beiden Seitenteilen sind scharfwinklig.

Der einzige vollkommen erhaltene Steinkern der R. Kl. (Fig. 5) gleicht in der Form der $V$. Dufrenoyi D'ORB, außerordentlich. Da von der Dreiteilung der Rippen nichts zu sehen, und die Selbständigkeit der Art nicht sicher ist, stelle ich das Exemplar vorläufig zu V. alata; andererseits ist zu bedenken, daß eine auf der äußeren Schale vorhandene Rippenteilung auf dem Steinkern nicht notwendig zum Ausdruck kommen muB.

Der deutlich sichtbare Schloßrand Fig. 7 zeigt eine flache, oben wie unten von einem schwachen Wulst eingefaßte Rinne, die vom Wirbel aus nach beiden Seiten an Breite abnimmt, mit engstehenden, ganz feinen, senkrecht zur Längserstreckung der Rinne gerichteten Zahnlamellen. 
Ein weiterer Steinkern stimmt in der Form vollkommen mit der V. curvicosta PHIL. (32), T. 18, Fig. 3, S. 31 überein. Das ganze Aussehen läßt gar keinen Zweifel darüber, daß wir es hier bloß mit einer verdrückten $\nabla$. alata zu tun haben, und hierauf stätzt sich meine Behauptung auf S. 276, daß V. curvicosta keine besondere Art ist.

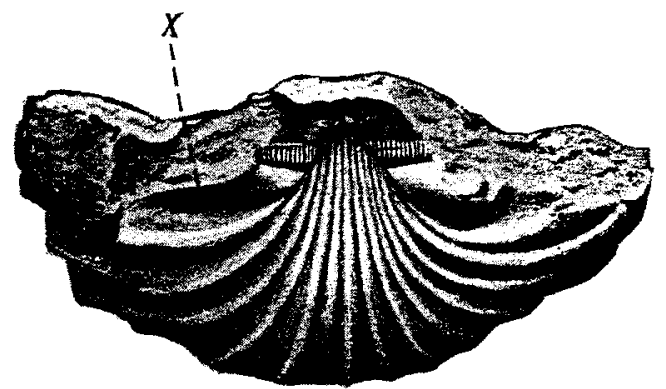

Fig. 7. Vola alata (v. Buch) Bayle et Coqu. Arroyo Blanco, Rio Atuel, Argentinien. Unt. Atuelsandstein des mittl. Lias. Steinkern der rechten Klappe, der die Schlobregion zeigt. Bei $x$ Furchen, welche Leisten entsprechen, die sich im Innern der Schale dort finden, wo das Ohr an den Hauptschalenteil stöBt.

\section{Abdrücke der linken Klappe}

2 Fragmente mit den im Vergleich zu den Zwischenräumen sehr schmalen Rippen, deren Rücken flach bis gerundet und mit scharfer konzentrischer Querverzierung.

Fundpunkte:

a) Argentinien: z. T. von Arroyo Blanco, Rio Atuel, z. T. auch von Milla Michi-Co.

b) Peru: ein Teil, und zwar das beste Material, von Chunumayo, andere von Ayasch.

c) Chile: Cordillere von Copiapo bei Amolanas und Manflas.

Vorkommen: In Südamerika in weitester Verbreitung in Peru, Argent. und Chile; hauptsächlich aus dem mittl. und ob. Lias, kommt allerdings auch bereits im unteren Lias vor. Näheres vergl. (10, S. 8), 80 , S. 20 ).

\section{V. alata (v. BUCH) BAYLE et COQU. var. Titan MöR.}

V. alata (v. Buch) BAYLE et Coqu. var. Titan Mör. (29), S. 41.

Pecten Titan Mör. PhIlispl (32), T. 15, S. 26.

Fragment einer außerordentlich großen Klappe. Rippen in der Wirbelgegend nur wenig breiter wie die Zwischenräume, während sie 
nahe dem Unterrand mindestens das dreifache der Breite der Zwischenräume erreichen. Sie treten in Wirbelnähe verhältnismäßig viel stärker hervor als in der Nähe des unteren Schalenrandes, besitzen einen flach gewölbten Rücken und fallen nahe dem Wirbel steil, nahe dem Unterrande flacher $z \mathfrak{z}$ den Zwischenräumen ab; Rippenflanken mit Längsfurchen und Riefen, die durch die Abwitterung entstanden sind.

Fundort. Neuquen.

Vorkommen: Von MöRICKE aus Chile ohne nähere Ortsangabe genannt. PHшIPPI nennt Jorquera (Cordillere von Copiapo). Bezüglich des Alters vergl. das bei der Stammform Gesagte; unser Exemplar aus dem unteren Lias.
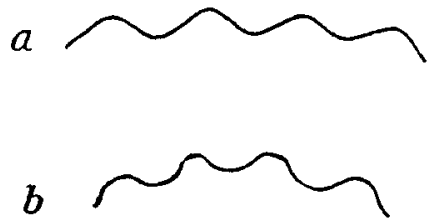

Fig. 8a. Vola Domeykoi PriL. Querschnitt durch die Rippen. Neuquen. Ont. Lias. Fig. 8b. Vola alata (v. BUCH) BAYIs et Coqu. Querschnitt durch die Rippen, die sich in ibrer Form der Domeykoi Parr. nähern. Lias der Cordillere von Copiapo. Chile.

\section{Domeykoi PHIL. Fig. 8.}

Pect. Domeykoi Pнic. (32), T. 13, Fig. 4.

"Dufrenoyi Phil. non D'Orb. Philippr (32), T. 13, Fig. 3, S. 24.

Ein Bruchstiick einer R. Kl. läßt nichts weiter als 9 Rippen erkennen, die vollkommen mit der PHILIPPI'schen Abbildung übereinstimmen. Der Querschnitt der Rippen gibt eine gleichmäßige Wellenlinie. Die Flanken der Rippen konvergieren firstartig nach dem Rücken, bilden aber keine scharfe Kante, sondern der Rücken ist gerundet, schmal. Boden der Zwischenräume schwach konkav, nicht eben. Flankenabfall der Rippen sanft geneigt, nicht steil bis senkrecht wie bei $V$. alata. Weiter ist nichts zu erkennen. Eine eigenartige Zwischenstellung zwischen dieser Spezies und der V. alata nimmt das bei V. alata beschrieb. Exempl. von Copiapó ein, das mit demselben Recht auch hier seinen Platz finden könnte.

Fundpunkt: Neuquen.

Vorkommen: Chile, Amolanas und Cordillere von Coquimbo. Lias, näheres nicht bekannt. In Argentinien neu im unteren Lias. 


\section{Bodenbenderi BeHr. Fig. 9 u. 10.}

7. Bodenbenderi BeHR. Behrevasen (3), T. 22, Fig. 3, S. 391.

V. aff. alata (v. Buch) Burckhardt, RoTh usw. (36). T. 4, Fig. 1-2, S. 20.

Das am besten erhaltene Stück (Fig. 9) gleicht der recht unvollkommenen BURCKHARDT'schen Abbildung außerordentlich. Die BEHRENDSEN'sche Abbildung stellt nur ein schlechtes Bruchstück der Wirbelregion dar. Es liegen vor: 4 beschalte Exemplare, 1 Steinkern.

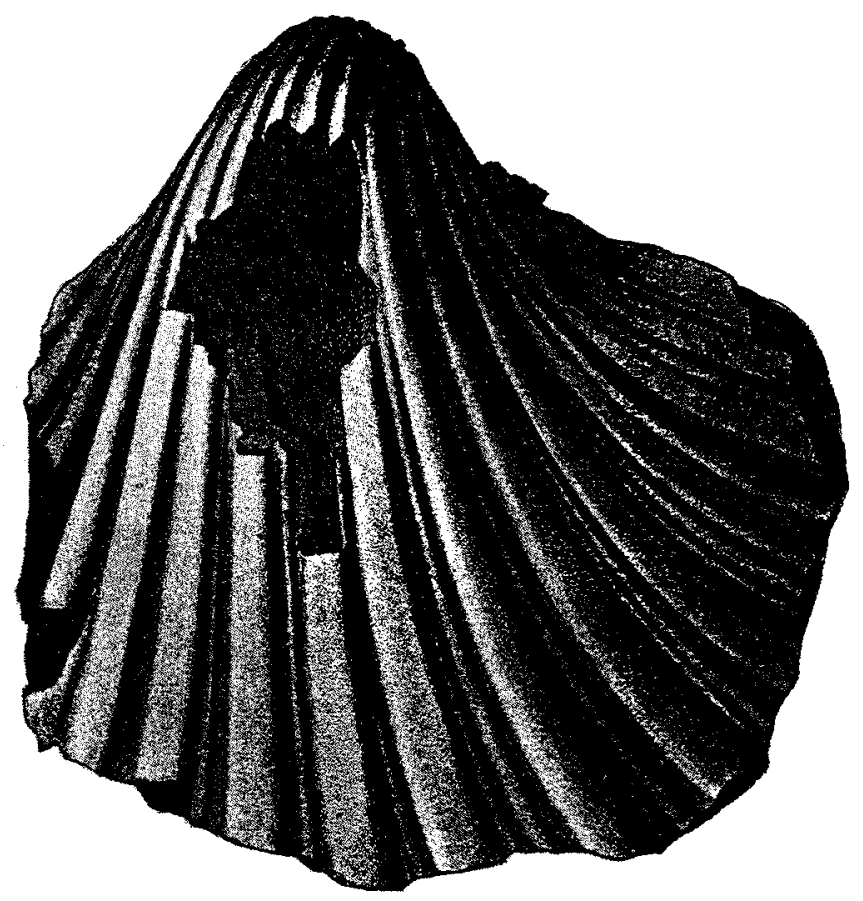

Fig. 9. Vola Bodenbenderi BerR. Piedra pintada. S.-Argentinien. Cardinien-Sch. des mittl. Lias. Rechte Klappe. $2 / 3$ nat. GröBe.

Rechte Klappe Fig. 9 mäßig stark gewölbt, Wirbel deutlich nach der Lk. Klappe zu eingekrümmt, aber nicht so stark wie bei $\nabla$. alata. 13-14 Hauptrippen, in der Wirbelnähe schmäler wie die Zwischenräume, mit ziemlich steilem Seitenabfall. Mit Annäherung an den Schalenunterrand werden die Rippen breiter wie die Zwischenräume, wobei die durch dichotome Spaltung entstehenden sekundären Rippen, die nach oben hin mit der Hauptrippe verfließen, zur Hauptrippe mitgerechnet sind. Infolge der dichotomen Zerspaltung der Hauptrippe erscheint 
nach dem unteren Schalenrand auch der Seitenabfall der Hauptrippen nicht mehr steil, sondern flach. In der Mitte der Zwischenräume e in e Zwischenrippe, die, in der Nähe des Unterrandes sehr deutlich, etwa $1 / 3-1 / 4$ so breit ist wie die Hauptrippe, mit Annäherung an den Wirbel schwächer wird und sich schließlich in einer Entfernung von $3-4 \mathrm{~cm}$ vom Wirbel ganz verliert. Die beiden letzten Flankenrippen sind nur durch einen ganz schmalen Zwischenraum getrennt, der keine Zwischenrippe trägt, und sind durch eine, bereits in unmittelbarer Nähe des Wirbels einsetzende Furche ziemlich genau in zwei gleiche Teile zerlegt, so daß diese letzten 2-3 Seitenrippen „eigentlich aus je einem Paar dicht nebeneinander liegender dünner Rippen" bestehen. Außer diesen eigentlichen $Z$ wischenrippen finden sich noch weitere Sekundärrippen, die aus den Hauptrippen durch dichotome Abspaltung an den Flanken derselben entstehen. Diese Zerlegung der Rippen ist bei den einzelnen Hauptrippen sehr verschieden weit vorgeschritten; an den dem Hinterrand genäherten. Rippen ist sie weniger ausgeprägt, wie in der Nähe des Vorderrandes. Die Abspaltung von Sekundärrippen erfolgt immer an der den Schalenflanken zugekehrten Seite der Rippen in stärkerem Maße, wie an der nach der Schalenmitte zu gelegenen. Die Bildung von Sekundärrippen ist also auf der Schale keineswegs überall gleich stark entwickelt. Die mediane Teilung der Flankenrippen ist auch eine solche dichotome Spaltung, die aber nicht wie auf dem mittl. Schalenteil an den Flanken, sondern auf der Mitte der Rippen einsetzt. Auf den Schalenflanken erscheint die Mittelrippe des Zwischenraums erst in größerer Entfernung vom Wirbel wie anf der Schalenmitte. Die dichotomen Spaltrippen setzen auf dem Flankenteil der Schale schon in größerer Wirbelnähe ein wie die Mittelrippe. Das Letztgenannte gilt besonders für den vord. Schalenteil. Anf dem hint. Teil der Schale sind die Mittelrippen viel schwächer oder auch noch gar nicht vorhanden, während die dichotome Abgliederung von Sekundärrippen schon gut erkembar ist. Letzterer Vorgang scheint sich also zuerst zu vollziehen und erst später die Bildung der Mittelrippe. Stellenweise sind feine konzentrische Anwachsstreifen erkennbar. Denken wir uns an unserem Exemplar wie bei dem von BEHRENDSEN nur die Wirbelgegend erhalten, so erhalten wir ein Bild, das mit der dortigen Beschreibung vollkommen übereinstimmt, insbesondere was die eigenartige Zweiteilung der Flankenrippen anlangt.

Linke Klappe (Fig. 10) deutlich konkav mit 13 Hauptrippen, die deutlich bis zum Wirbel durchziehen und von der Mitte der Schale nach beiden Flanken an Breite zu- und an Höhe abnehmen. In der 
Mitte des Schalenunterrandes, etwa $1 / 2$ so breit wie die Zwischenräume, erreichen sie auf den Flanken schließlich mindestens die Breite derselben. Sie sind flacher wie die Rippen der R. Kl. Zwischenräume schwach konkav mit deutlicher Mittelrippe, die auf der Mitte der Schale und in der Nähe des Unterrandes etwa $1 / 3$ bis $1 / 4$ der Breite des Intercostalraumes erreicht. Vom Wirbel nach dem Unterrand nimmt die Zwischenrippe stärker an Breite zu wie der Zwischenraum. Je mehr wir uns

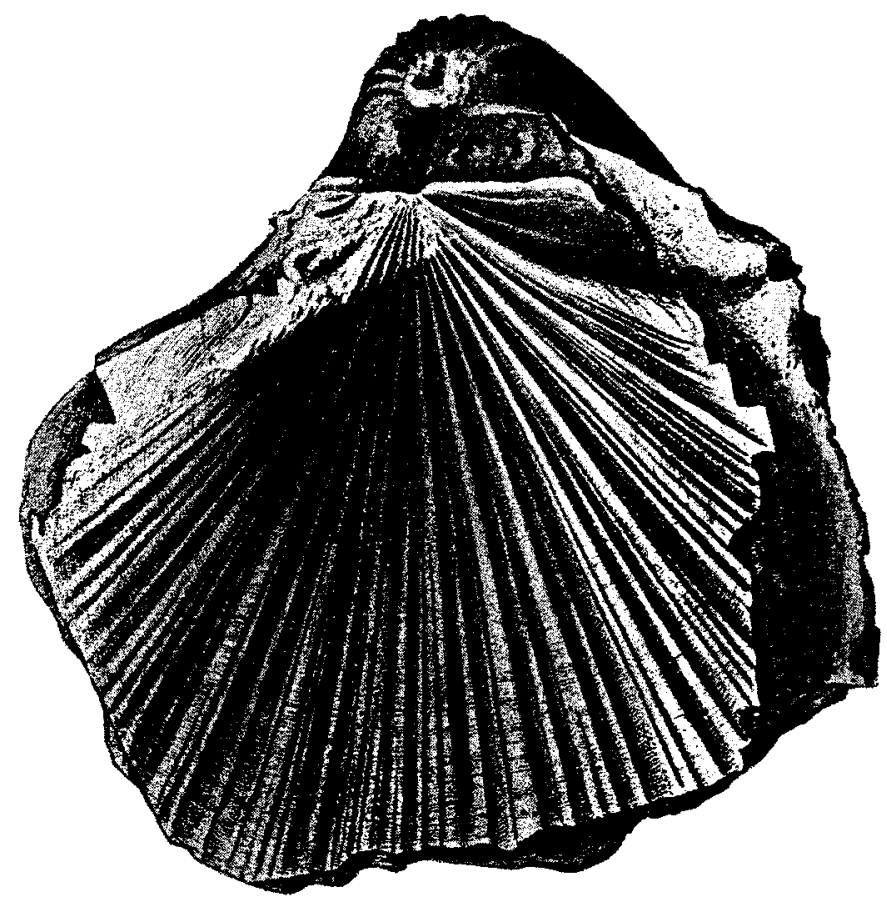

Fig. 10. Dola Bodenbenderi BEHR. Piedra pintadra, Argentinien. Cardinien-Sch. des mittl. Lias. Linke Klappe. $2 / 3$ nat. Größe.

der Schalenmitte nähern, um so weiter lassen sich die Zwischenrippen zum Wirbel hin verfolgen, genau wie auf der R. Kl. Außer den Zwischenrippen begleiten die Flanken der Hauptrippen noch Spaltrippen, die durch dichotome Zerteilung der letzteren entstehen. Die Spaltrippen sind auf dem Schalenvorderteil stärker und fortgeschrittener ausgebildet wie auf dem hinteren Teil, in Analogie mit der R. Kl. Zahl der Spaltrippen einer Hauptrippe nimmt von der Schalenmitte nach den beiden Flanken zu. Mehr wie 2 Spaltrippen an der Seite einer Hauptrippe werden nicht ausgebildet. Die Spaltrippen bilden sich stärker auf 
der der Schalenmitte zugewandten Seite der Rippen aus, wie auf der nach den Schalenflanken zu gelegenen, also das Umgekehrte, wie auf der R. Kl. Sind 2 Spaltrippen auf einer Seite der Hauptrippe vorhanden, so entsteht die der Mittelrippe des Zwischenraums am nächsten gelegene erst in größerer Entfernung vom Wirbel wie die andere. Die Skulptur ist im ganzen schon viel ausgeprägter und komplizierter wie auf der R. Kl. Rippen wie Zwischenräume in gleicher Weise mit einer sehr feinen, konzentrischen Anwachsstreifung. Hinter der letzten Flankenrippe auf beiden Flanken ein paar feine rippenartige Linien, die den Rippen parallel verlaufen. Das hint. Ohr ziemlich groß; es reicht über die Mitte der hint. Schalenhälfte hinaus und zeigt feine, etwas welliggebogene Anwachslinien und radial vom Wirbel ausstrahlende Rippen. Zwei stärkere Radialrippen deutlich erkennbar, zwischen die sich vielleicht (?) noch kleinere, aber nicht sicher erkennbare, einschalten. Vord. Ohr unbekannt.

Innenseite der Lk. Kl. mit sehr flachen, nur wenig hervortretenden Rippen und Zwischenräumen, die als schnurgerade Streifen vom Wirbel nach dem Unterrand verlaufen. Rippen schmäler wie die Zwischenräume etwa $1: 2$. Die den Rippen der Außenseite entsprechenden Intercostalräume der Innenseite mit sekundären Rippen und Furchen, welche den Spaltrippen der Außenseite entsprechen und bis ins kleinste deren ausführlich besprochene Anordnung erkennen lassen, worauf sich hauptsächlich die Bestimmung stützt. Die Zwischenrippe der Außenseite innen nicht ausgeprägt.

Hierhin stelle ich mit Vorbehalt 2 weitere Exemplare, welche auch nur die Innenansicht zeigen; vielleicht auch einen Steinkern von Arr. Blanco, der im allgemeinen mit dem Alata-Steinkern übereinstimmt, aber Furchen auf den Rippen beider Klappen zeigt. (Bildung von Spaltrippen??)

Fundpunkt: Das abgebildete Stück und der Innenabdruck der Lk. Kl. von Piedra pintada. Die anderen alle mehr oder weniger zweifelhaften Stücke von Perú (Ayasch) und Arr. Blanco.

Vorkommen: Bis jetzt nur aus Argentinien von Portezuelo ancho und Piedra pintada bekannt aus dem mittl. bezw. unt. (?) Lias. Meine Exemplare aus mittl. Lias.. 


\section{B. Pectiniden der Pradoanus-Gruppe}

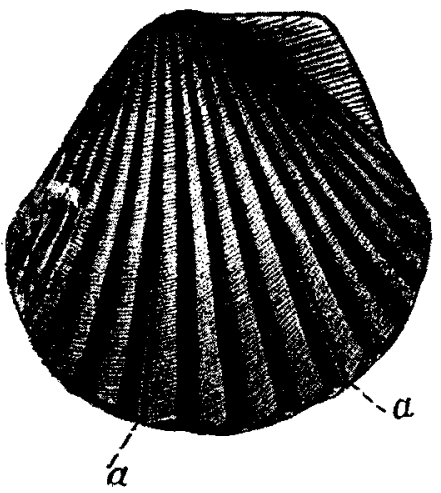

Fig. 11 a.

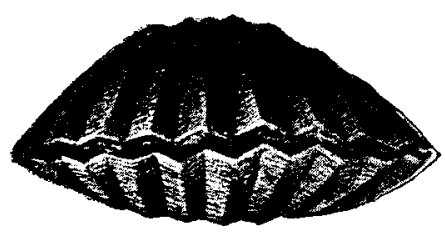

Fig. $11 \mathrm{~b}$.

Pecten Pradoanus Vern. et Coltomb. Chunumayo, N.-Peru. Alata-Kalk des unteren Lias.

a Rechte Klappe von oben gesehen.

$b$ Beide Klappen gegen den Stirnrand gesehen.

Pecten Pradoanus VernediL et CoLL. Fig. 11a u. b.

P. Pradoanus Vern. et Coli. Benrendsen (3), T. 22, Fig. 1 , S. 39.

$" \quad " \quad " \quad$ Vernevil et Coll. (43), T. 3, Fig. 4, S. 163.

\begin{tabular}{|c|c|c|c|c|c|c|}
\hline & & & I & II & III & IV \\
\hline Höhe & 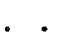 & 38 & $\mathrm{~mm}$ & $37 \mathrm{~mm}$ & $36 \mathrm{~mm}$ & $40 \mathrm{~mm}$ \\
\hline Länge & & 36 & $"$ & $36 "$ & $35 n$ & $43 n$ \\
\hline Dicke & . & 20 & $n$ & & & \\
\hline
\end{tabular}

Die Exemplare stimmen mit den Abbildungen and Beschreibungen von BeHRENDSEN und VERNEUIL et Collomb überein. Beide Klappen, von der stets geringeren Wölbung der Lk. Kl. abgesehen, sind ganz gleich. Die Lk., schwächer gewölbte Kl. kann nach VERNEUL et CoLLOMB flach werden, wird aber niemals konkav. 13-14'scharfe, dreieckige Rippen, durch ebensolche Zwischenräume getrennt, zeigen deutliche, linienfeine bis feinlamellöse Anwachsverzierung, die in der gleichen Weise über Rippen und Zwischenräume fortsetzt. Auf dem untersten Teil des Seitenabfalles der Rippen findet sich ein feiner linienartiger Walst (Abd. 11 a bei $a$ ), und der Grund der Zwischenräume erscheint furchenartig eingetieft, was besonders auf dem Steinkern hervortritt. Das Ohr der Lk. Kl. (wohl auch der R.) ist mit schräg verlaufenden Rippen bedeckt, die sich in der gleichen Weise auf dem zwischen dem Ohr und der letzten Rippe liegenden Flankenteil der Schale finden. Das vord. Ohr, an dem ein etwaiger 
Byssusausschnitt zu suchen wäre, ist leider zerstört. Auch BEHRENDSEN drückt sich ungewiß aus, ,an welchen ein Byssusausschnitt nicht dentlich erkennbar ist". Der Steinkern ist, wie BEHRENDSEN hervorhob, von der Außenseite der Schale grundverschieden, mit gerundeten, die halbe Breite der Zwischenräume erreichenden Rippen. Ein kleines Exemplar paßt gut zu der BEHRENDSEN'schen Abd. Er gleicht dem. Steinkern der V. alata (v. BucH) BAYLE et COQU. so sehr, daß ich auf dessen Beschreibung und Abbildung, S. 281, verweisen kann, auch hinsichtlich der Einzelheiten des Schloßbaues. Ich könnte sogar den Steinkern nicht von $V$. alata trennen, wenn er sich nicht mit dem zugehörigen Abdruck zusammengefunden hätte, der scharfe dreieckige Rippen zeigt. Außerdem ist die Wölbung geringer wie bei $V$. alata. Auf dem Steinkern sind die Ohren nicht berippt, es ist also diese Skulptur auf die Außenseite beschränkt.

Fundort: Neuquen, Milla Michi-Có in Argent.; Chunumayo in Perú. Vorkommen:

a) In Europa: im mittleren Lias von Spanien.

b) In Südamerika: bis jetzt nur aus dem mittleren Lias von Portezuelo ancho. In Perú und S.-Argentinien neu; unterer Lias.

\section{Pecten cardioides PHIL.}

P. cardioides PhIL. Philtppi (32), T. 17, Fig. 2, S. 29.

Hierhin gehört ein Bruchstück eines Abdruckes, das außergewöhnlich scharfe, dreieckige Rippen, sehr stark gewölbte Form und einen stark eingekrümmten Wirbel zeigt. Wegen der starken Einkrümmung des Wirbels halte ich das Stück für eine R. Kl. Die ganz erhaltene Wirbelregion läßt 12 messerscharfe Rippen erkennen. Anwachsstreifẹn sind nur an einer Stelle angedeutet. Das hintere Ohr zeigt nichts von den bei PHسLPPI erwähnten schrägen Linien, was ich mir, ebenso wie das Fehlen der Anwachsverzierung, durch den Erhaltungszustand als Abdruck erkläre. Der Steinkern zeigt in der Wirbelgegend genau dieselbe Schloßbildung wie bei $V$. alata und Pect. Pradoanus. Überhaupt gleicht das Stück der V. alata außerordentlich; für die Unterscheidung maßgebend sind die sehr scharfen Rippen, die auch PHuIPPI bei den zahlreichen, der $V$. alata nahestehenden Arten, sonst nirgends kennt. Andererseits ist das Stück dem Pect. Pradoanus sehr ähnlich, von dem es nur durch seine Größe direkt zu unterscheiden ist.

Hierhin gehören vielleicht auch noch einige fragmentäre Abdrücke mit scharfen, großen, dreieckigen Rippen, die für V.alata zu scharf, und 
für Pect. Pradoanus za groß sind und aus denen sich, die Richtigkeit der Bestimmung vorausgesetzt, für Pect. cardioides die gleiche Beschaffenheit des Schaleninnern wie bei $V$. alata herleiten läßt.

Fundort: Milla Michi-Có.

Vorkommen: Von PHILIPPI aus dem chilenischen Lias ohne nähere Altersangaben beschrieben. In Argentinien im mittleren Lias.

\section{Teil. Zur Stammesgeschichte der Gattung Vola}

A. Gliederung der Lias-Volen Südamerikas

Unter den Volen des südamerikanischen Lias kann man 2 Hauptgruppen unterscheiden:

Gruppe I: Fig. 1-7 Hauptrippen und Zwischenräume der R. Kl. niemals mit Sekundärrippen, stets einfach. Rippen der Lk. Kl. bisweilen mit feinen Furchen und Sekundärrippen, die Zwischenräume aber stets einfach, niemals eine deutliche $Z$ wischenrippe aufweisend. Rippen im allgemeinen schmäler wie die Zwischenräume. Wirbel der R. Kl. stark eingekrümmt. Typus: V. alata (V. BUCH) BAYLE et COQU.

Gruppe II: Fig. 9-10 Hauptrippen beider Klappen mit mehreren deutlichen Spaltrippen oder wenigstens Andeutung zur Ausbildung solcher. In den Zwischenräumen beider Klappen deutliche Zwischenrippen. Wirbel der R. Kl. weniger stark eingekrümmt. Typus: V. Bodenbenderi BEHR.

In der Gruppe I lassen sich zwei weitere Unterabteilungen unterscheiden.

Abteilung A. Rippen der R. Kl. mit steil, fast senkrecht zu den Zwischenräumen abfallenden Flanken, aber sonst recht verschieden ausgebildet. Rücken breit oder schmal, eben oder schwach konvex, aber immer deutlich gegen die Flanken abgesetzt, niemals langsam in dieselben übergehend, derart, daß Rippen und Zwischenräume im Querschnitt das Bild einer Wellenlinie ergeben, vielmehr im Querschnitt einem Zinnenkranz gleichend (Fig. 1 n. 3). Typus: V. alata (v. BUCH) BAYLE et COQU.

Abteilung B. Rippen der R. Kl. mit den Flanken sąnft nach den Zwischenräumen abfallend, Rücken schmal and gerundet. Rippenflanken winklig nach dem Rücken konvergierend (der aber, soweit mein Material und PHEIPPI's Abbildung (32) das zeigen, niemals als scharfe Kante entwickelt, sondern immer gerundet ist). Querschnitt der Rippen und Zwischenräume das Bild einer regelmäBigen Wellenlinie 
ergebend. Vergl. Fig. 8a und Fig. 8b; (32) T. 13, Fig. 3-4. Typus: V. Domeykoi PHIL.

Zwischen A und B existieren Übergänge, wie das chilenische Exemplar von V. alata Fig. $8 \mathrm{~b}$.

B. Beziehungen der Lias-Volen Südamerikas zu jüngeren Volen und Pectiniden

Die Gattung Vola tritt im Lias Ssüdamerikas zuerst auf, fehlt im ganzen jüngeren Jura und findet sich erst wieder in der unteren, häufiger in der oberen Kreide, und vor allem mit vielen Arten im Tertiär und in der Gegenwart. Über den phylogenetischen Zusammenhang der Vola-Formen der genannten Formationen sind verschiedene Ansichten ausgesprochen worden. Einige Autoren, wie z. B. MEYERExMar (28) S. 16 haben die enge Zusammengehörigkeit und Unteilbarkeit der Gattung Janira betont. Andere, wie Doणv世LE (20) S. 202-205 haben behauptet, daß speziell die Kreide- and Tertiär-Janiren in keinem Zusammenhang miteinander stehen, sondern daß es sich um analoge Umbildungen verschiedener Gruppen des Pecten-Stammes handelt, nehmen also Konvergenzbildung an. „Ich (PHILIPPI) schließe mich letzteren Forschern an ...." (31) S. 111. Ich habe daraufhin meine Liasformen eingehend mit jüngeren, besonders tertiären und rezenten Vola-Formen verglichen und bin zu der Ansicht gekommen, daß sich auf Grund des vorliegenden Tatsachenmaterials mit besserer Begründung wie PHILIPPI's Ansicht, die Auffassung eines direkten phylogenetischen Zusammenhanges der Lias- und Tertiär-Volen vertreten läBt. Die Begründung dieser Behauptung sei im folgenden näher ausgeführt:

\section{Eozän-Janiren}

Die ältesten Tertiär-Volen treten nicht, wie PHInIPPI sagt, erst im Oligozän, sondern, nach SACCO, bereits im Eozän auf: V. (Pecten) Michelotti D'ARCH. (15) T. 12, Fig. 20-21, S. $435=V$. (Pecten) arcuata var. Michelotti D'ARCH. SACCO (37) S. 67. DÉPÊRET et ROMAN (16) halten das gleiche Vorkommen allerdings auch für Oligozän. Die stark gewölbte R. Kl. trägt 20-22 gleiche, gerundete, durch engere Zwischenräume getrennte Rippen, die zuweilen in Wirbelnähe durch eine Furche geteilt sind. Das gleiche Merkmal heben BURMEISTER \& GIEBEL bei $\nabla$. alata hervor und erklären es durch Verwitterung. Der SchloBrand trägt ${ }_{n}$ de stries pectiniformes très serrés" $(\mathbf{1 5})$, also die gleichen Zähnchen wie bei 
V. alata. Die konkave Lk. Kl. trägt die gleiche Anzahl Rippen, doch sind sie schmäler als die Zwischenräume, anscheinend sogar dreieckig. Beide Klappen zeigen in den Zwischenräumen feinschuppige Querverzierung wie $V$. alata, nur stärker ausgebildet. Schon bei dieser EozänForm finden sich also mehrere Merkmale wieder, die stark an die Liasform erinnern. Die abweichende Größe kann man hier wie auch bei den folgenden Formen nicht als wesentlichen Unterschied bezeichnen.

\section{Oligozän-Janiren}

Im Oligozän tritt Vola reichlicher auf mit der Areuatus-Gruppe (nach V. (Pecten) arcuata BRocchI (5) T. 14, Fig. 11 benannt). Schon PHILIPPI hebt die große Ähnlichkeit mit den Liasformen hervor. Die stark gewölbte R. Kl. zeigt breite, gerundete Rippen, breiter als die Zwischenräume, die konkave Lk. solche, die schmäler sind als die Zwischenräume, auch ist eine feinschuppige Querverzierang vorhanden: alles Merkmale, die aufs genaueste mit $\nabla$. alata übereinstimmen. Ihnen gegenüber fällt die größere Zahl der Rippen (20) und die geringere Größe nicht ins Gewicht. Ersterer Unterschied darf bei der großen Zeitlücke Lias-Oligozän kein Wunder nehmen, und der Größenunterschied kann sehr leicht durch andere, vielleicht weniger günstige Lebensbedingungen seine Erklärung finden. Der normale Pect. arcuatus (37) T. 21, Fig. 14-30, S. 65 hat in der R. Kl. breite, gerundete Rippen wie die Abteilung IA des Lias ${ }^{1}$ ). Gleichzeitig ist auch eine Varietät von Pect. arcuatus bekannt, die V. arcuata BROC. var. angulocostata SACCO (37) T. 21, Fig. 32-34, deren Rippen auf dem Rücken nicht breit, sondern scharf sind (S. 66) „costae radiales perspicuae, angulate", mit winklig abfallenden Seiten, die der Abteilung IB des Lias entsprechen würde. SACCOS Abd. ist allerdings so mangelhaft, daß sie nicht viel erkennen läßt. Beide Formen stammen aus dem Tongérien. Auch die Gruppe II des Lias mit sekundär geteilten Rippen finden wir im Oligozän wieder in Gestalt der V. arcuata BROccHi var. deperdita Michenotri (37) T. 21, Fig. 35-36, bei der, wie auch PH世IPPI hervorhebt, Sekundärberippung auftritt. Das Gleiche gilt auch von der von PHEIPPI zitierten V. (Pecten) rupeliensis vON KOENEN aus dem Mittel-Oligozän.

1) Der Kürze wegen werde ich mich zur Bezeichnung der verschiedenen Gruppen im Text der denselben auf S. 291 vorangesetzten Zahlen and Buchstaben bedienen. Weiterhin möchte ich zur gröBeren Klarheit die Gruppen nach ihren typischen Vertretern bezeichnen: Gruppe I = Alata-Gruppe, Gruppe II = Bodenbenderi-Jacobaeus-Gruppe. Abteil. A = Alata-Groppe s. str., Abteil. B = Domeykoi-Gruppe. 


\section{Miozäne und postmiozäne Janiven}

Im Miozän und in jüngerer Zeit finden wir eine sehr reichliche Entwicklung von Vola, deren neogene Vertreter Europas von DËPÊRETROMAN in der des öfteren zitierten Arbeit (16) monographisch bearbeitet sind $^{1}$ ). Ich werde der Übersichtlichkeit wegen weiterhin den Alata- und Bodenbenderi-Jacobaeus-Stamm getrennt verfolgen.

\section{Gruppe II: der Bodenbenderi-Stamm}

Ganz ähnliche Verhältnisse wie $V$. Bodenbenderi BEHR. aus dem Lias zeigt die $V$. (Pecten) grandis Sow. ${ }^{2}$ ) aus dem Pliozän des Nordatlantischen Meeres (England, Belgien). Am besten tritt das auf den Abbildungen (30) a. (40) hervor. Beide zeigen auf beiden Klappen Hauptrippen, die sekundär geteilt sind, und in den Zwischenräumen Zwischenrippen. Es sind 13 Rippen vorhanden (nach NYST mehr), die auf der konvexen Schale stärker sind als auf der konkaven, auf der ersteren breiter als auf der letzteren, sowohl untereinander verglichen; wie auch im Verhältnis zu den Zwischenräumen. Die konzentrische Anwachsverzierung ist auf der konvexen $\mathrm{Kl}$. weniger deutlich wie auf der konkaven, „they are very regular, close and raised into sharp laminae" (40). Die Ohren der Lk. Kl. tragen radial vom Wirbel ausstrahlende Rippen, die weiter entfernt stehen, und sehr feine engstehende Anwachsstreifen. In allen diesen Merkmalen stimmt $V$. Bodenbenderi nit $V$. grandis überein. Der einzige Unterschied ist eigentlich nur der, daß bei der Liasform die Sekundärberippung; besonders auf der R. Kl., noch nicht so vollkommen durchgeführt ist, sondern sich noch mehr in einem Anfangsstadium befindet. Bei $V$. Bodenbenderi ist der Wirbel schwächer eingekrümmt als bei den Formen des Alata-Stammes. Das Gleiche ist bei V. grandis gegenüber den tertiären Vertretern des Alata-Stammes der Fall, wie wir noch sehen werden.

Eine andere jungtertiäre Form, die mit der V. Bodenbenderi aus dem Lias viele Ähnlichkeit hat, dementsprechend, wie auch schon DEPERET (16) Suppl. S. 100-101, Fig. 42 betont, der V. (Pecten) grandis Sow. nahesteht, ist $\nabla$. (Pecten) Vasseli Fuchs (4) S. 185. Die mäßig stark konvexe $\mathrm{R} . \mathrm{Kl}$. hat 11-12 Hauptrippen, welche der Länge nach durch eine

3) Eine Kritik dieser Arbeit findet sich in "Die Vola-Arten des ägyptischen und syrischen Neogen" von BlaneenhorN (4). Die den einzelnen Spezies auf den folgenden Seiten beigegebenen Diagnosen sind meistens der DrPERET'schen Arbeit entnommen.

2) (16) T. 8, Fig. 3, 3a, S. $61-62$; (40) Bd. 6, 'T. 585, S. 163; (30) T. 21, Fig. 6, T. 22, Fig. 1, S. 284. 
Furche in Sekundärrippen erster Ordnung geteilt sind, und diese sind jeweils nochmals durch eine erst in der halben Entfernung vom Wirbel zum Schalenrand einsetzende Furche in 2 Sekundärrippen zweiter Ordnung zerlegt. Wir haben also eine zweimalige dichotome Teilung der Hauptrippen der R. Kl., wie sie bei $V$. Bodenbenderi stellenweise auch bereits angedeutet, wenn auch noch nicht durchgeführt ist. Auf beiden Flanken liegen 2-3 einfache Rippen. In den Zwischenräumen, die schmäler sind als die Rippen, finden sich „quelques costulles intercalaires irregulièrement disposées". Die Zwischenrippen sind unregelmäßig verteilt, nicht überall vorhanden, in schönster Übereinstimmung mit der Liasform. Der Wirbel ist mäBig stark eingekrümmt, wie bei $V$. grandis. Die Lk. Kl. trägt 10-11 Rippen, die schmäler als die Zwischenräume und in der Nähe des Unterrandes dichotom zerteilt sind, zuweilen aber auch einfach bleiben, also wieder die unregelmäßige Verteilung der Sekundärrippen zeigen wie bei der Liasform, in einem gewissen Gegensatz zu $V$. grandis. Es findet sich eine ziemlich starke Zwischenrippe, die bis in die Wirbelgegend reicht; zuweilen sind zwischen Haupt- und Zwischenrippe in der Nähe des Unterrandes noch weitere Zwischenrippen eingeschoben. Die Ohren tragen nur wenige Rippen und feine Anwachsstreifen. Wir sehen also eine weitgehende Übereinstimmung zwischen $V$. Vasseli und $V$. Bodenbenderi. Die ganzen Sekundärrippen bei der Tertiärform sind nichts weiteres wie die stärkere Ausbildung des bereits bei der Liasform vorhandenen Typus, und besonders charakteristisch ist, daß auch noch bei $V$. Vasseli eine gewisse Unruhe, Unregelmäßigkeit in der Verteilung der sekundären Elemente zum Ausdruck kommt wie bei $V$. Bodenbenderi, während das bei $V$.grandis nicht mehr so sehr der Fall ist. Die SowERBY'sche Abbildung von $V$. grandis gleicht der $\nabla$. Vasseli erheblich mehr wie die DePERET'sche. Vorkommen: ob. Pliozän in der Gegend des Roten Meeres, Suez. Es ist mir nicht entgangen, da $B$ zwischen $\nabla$. grandis und $V$. Vasseli' ein gewisser Unterschied besteht, den schon DEPERET hervorhebt. Bei V. grandis sind die Rippen der R. Kl. bloß von ziemlich tiefen Furchen durchzogen, während sie bei $V$. Vasseli direkt zweimal dichotom zerteilt sind. Dieser Unterschied erscheint mir aber nur als ein gradueller: wenn wir uns bei $V$. grandis die Furche noch tiefer in die Hauptrippen eingesenkt vorstellen, so erhalten wir auch die dichotome Zerteilung. Aus dem noch unvollkommenen, noch in der Umbildung begriffenen Stadium der R. Kl. von V. Bodenbenderi können beide Arten von sekundärer Gliederung der Rippen entstanden sein. 
Tertiäre Pectiniden mit Beziehungen zu den Volen des Bodenbenderi-Stammes

Auch unter den echten Pectiniden des Tertiär mit 2 gewölbten Klappen finden sich Formen, die in manchem an unseren liasischen Bodenbenderi-Stamm erinnern, wie Patinopecten (Pecten) Oweni ARNotD (1) T. 8, Fig. 1, S. 63 aus Miozän u. Pliozän Kaliforniens. Die Lk. Kl. ist viel schwächer konvex als die R. Letztere hat 15-16, den Zwischenräumen an Breite gleiche Hauptrippen und in den Zwischenräumen dentliche Zwischenrippen. Die Hauptrippen sind in einiger Entfernung vom Wirbel durch eine Furche mehr oder weniger deutlich dichotom geteilt, wie dies bei der Liasform auch angedeutet, doch ist die Teilung etwas weiter vorgeschritten, aber nicht so weit wie bei $\nabla$. grandis Sow. Die Hauptrippen der Lk. Kl. sind schmäler als die Zwischenräume; letztere zeigen eine Zwischenrippe; die Hauptrippen sind durch Furchen in verschieden starkem Maße in Sekundärrippen zerteilt. Die Ohren haben wenige weitstehende Radialrippen und feine dichtgedrängte Anwachsstreifen. Beide Kl. tragen konzentrische Anwachsverzierung, die anscheinend auf der $R$. schwächer ist wie auf der Lk. In allen diesen Punkten stimmt Patinopecten Oweni mit Vola Bodenbenderi und dementsprechend auch mit $V$. grandis und Vasseli überein; der Unterschied liegt nur in der schwachen Konvexität der Lk. KI. und einem deutlichen Byssusausschnitt des vord. Ohres bei der kalifornischen Form.

\section{Gruppe I: der Alata-Stamm}

Die miozäne Subarcuatus-Gruppe von DĖPERET (16) S. 10-17 steht der oligozänen Arcuatus-Gruppe sehr nahe, und es besteht zwischen beiden kein wesentlicher Unterschied. Wir finden auch hier die Vertreter der beiden liasischen Gruppen wieder: Abteil. $\mathrm{A}=$ Alata-Stamm s. str.: Rippen oben flach, mehr oder weniger breit, mit steil abfallenden Seiten; Abteil. B = Domeykoi-Stamm: Rippen mit sanft abfallenden, nach oben konvergierenden Flanken, Rücken mehr oder weniger gerundet.

\section{Abteilung A: Alata-Stamm s. str.}

Pecten subarcuatus TouRNoukR (16) Fig. 17, S. 38 besitzt auf der R., stark gewölbten Kl. 18-19 auf dem Rücken breit gerundete bis flache Rippen, schmäler wie die Zwischenräume. Die Lk. konkave Kl. hat scharfe, dreieckige Rippen in der gleichen Zahl. Die feinen Anwachslamellen sind auf der Lk. Kl. und in den Zwischenränmen schärfer 
ausgebildet wie auf der R. Kl. und auf den Rippen. Vorkommen: Miozän, bes. Helvetien. Gleichfalls der V. alata sehr ähnlich sind die außerhalb der DEPERET'schen Subarcuatus-Gruppe stehenden: V. (Pecten) urmiensis ABICH und $V$. (Pecten) convexocostatus ABICH. $\nabla$. (Pecten) urmiensis ${ }^{1}$ ): die R. stark gewölbte Kl. hat $16-20$ untereinander gleiche, rechteckige Rippen, die breiter sind als die Zwischenräume. Der Wirbel ist stark eingekrümmt. Die Rippen treten scharf hervor und liegen wie bei $V$. alata "wie Leisten auf der Schale“. Die Zwischenräume tragen konzentrische Anwachslamellen. Daß die Rippen breiter sind als die Zwischenräume, ist nicht wichtig, da dieses Verhältnis, wie schon hervorgehoben, auch bei $V$. alata schwankt. Einen wesentlichen Unterschied gegenüber der Liasform kann ich nicht angeben. Die Form liegt in der I. Mediterranstufe in Persien und Russisch-Rumänien. Das gleiche gilt von $V$. (Pecten) convexocostatus ABICH (16) T. 6, Fig. 7, S. 55, mit stark gewölbter R. Kl. und stark eingekrümmtem Wirbel; die R. Kl. hat hohe, subquadratische Rippen, breiter wie die Zwischenräume, die keine konzentrischen Anwachslamellen zeigen. Die Lk. Kl. ist unbekannt. Vorkommen: Armenische Region, I. Mediterran-Stufe.

\section{Abteilung B: Domeykoi-Stamm}

Auch in der Subarcuata-Gruppe sind Formen vertreten, die eine der liasischen Domeykoi-Gruppe analoge Rippenbildung aufweisen, also zu den bis jetzt beschriebenen neogenen Volen in demselben Verhältnis stehen, wie im Lias die Alata-Gruppe s. str. zn der Domeykoi-Gruppe. V. (Peeten) cristatocostata SACco (16) T. I, Fig. 11-12, S. 14. Die stark gewölbte R. Kl. hat 18-20 enge, dreieckige Rippen, die bei DEPERET scharfrückig sind. Alata-Formen mit ganz scharfen Rippen der R. Kl. sind mir allerdings unbekannt; doch ist demgegenüber hervorzuheben, daß auf den Abbildungen (23) T. 8, Fig. 1-6 die Rippen oben nicht scharf, sondern wie bei $V$. Domeykoi sanft gerundet erscheinen. Die Rippen sind gleich breit wie die Zwischenräume, die feine konzentrische Anwachsverzierungen tragen. Die Lk. Kl. hatt 16-17 scharfe, dreieckige Rippen und konzentrische Anwachsstreifen in den Zwischenräumen. Vorkommen: II. Mediterranstufe, besonders Helvetien; Circummediterrangebiet und Portugal (Lissabon). Eine Zwischenstellung zwischen Pect. subarcuatus BROCCHI und Pect. cristatocostatus SACCO in bezug auf Berippung nimmt $V$. (Pecten) Fuchsi FonTanNes ein (16) T. 1, Fig. 5-10,

1) Da bei beiden Formen die Berippung der Lk. Kl. unbekannt ist, so muB ich es dahingestellt sein lassen, ob sie hierhin gehören oder zu der später zu besprechenden Gruppe mit breiten Rippen in der Lk. Kl. 
S. 14, wie schon DEPERET hervorhebt. Ganz analog wie wir im Lias eine Zwischenform zwischen der Abteilung A und B hatten (S. 292), so tritt nns hier eine solche in der Gestalt des Pect. Fuchsi FonT. entgegen. Vorkommen: II. Mediterranstufe, besonders Helvetien; Circummediterrangebiet und Portugal (Lissabon).

Sehen wir von der Arcuata-Gruppe ab, so finden sich weiterhin unter den Tertiän-Janiren-Formen, die sich ebenfalls auf die Typen der beiden Lias-Abteilungen verteilen lassen, sich aber von ihnen gemeinsam dadurch unterscheiden, daß die Rippen der Lk. Kl. nicht wie bei $V$. alata mehr oder weniger scharf sind, sondern flach, von wechselnder Breite, die indessen im allg. hinter der der Zwischenräume zurücksteht. Mein Material weist keine Liasformen mit typisch flachen breiten Rippen in der Lk. Kl. auf, wenn auch bei einem Stück die Rippen desselben mit Annäherung an den Unterrand sich sichtlich verflachen und verbreitern. Ich muß es also dahingestellt sein lassen, ob Formen, deren Rippen in der Lk. Kl. einen deutlich flachen, ebenen Rücken haben, schon zur Liaszeit vorkommen oder erst in jüngerer Zeit aus scharf berippten entstanden sind.

Formen vom Typus der Alata-Gruppes. str. mit flachen, breiten Rippen in der Lk. Kl.

V. (Pecten) Josslingi Sмштн (16) T. 5, Fig. 3-5, S. 41. Die R. Kl. hat 10-11 subquadratische Hauptrippen, durch viel engere $Z$ wischenräume getrennt, und jederseits 3-4 kleinere, etwas abweichende Flankenrippen; sie ist stark gewölbt, mit stark eingebogenem Wirbel. Es dürfte schwer sein, einen wesentlichen Unterschied gegenüber $V$. alata anzugeben. Die 12-13 quadratischen Rippen der Lk. Kl. sind schmäler als die Zwischenräume, die eine deutliche konzentrische Anwachssteigerung zeigen. Circummediterrangebiet, Frankreich und Portugal (Lisssabon). Vindobonien.

V. (Pecten) Tietzi Fuchs (16) T. 2, Fig. 5, S. 22 steht der vorhergehenden Spezies außerordentlich nahe, von der sie sich, nach DEPERET, nur durch die zahlreicheren und engeren Rippen unterscheidet. Interessant ist, daß dieser Forscher schon auf die groBe Ähnlichkeit mit $P$. arcuatus BROCCHI und deperditus MICHEL. hinweist.

V. (Pecten) Kochi LOCARD (16) T. 2, Fig. 5, S. 23, (4) S. 172 hat 15-18 fast quadratische Rippen in der R. Kl., etwas breiter wie die Zwischenräume. Letztere tragen Anwachslamellen, welche in dem Intercostalraum zweimal gebuchtet erscheinen, worin DEPERET den Beginn der Sekundärrippenbildung sieht. Die Lk. Kl. hat 14-16 Rippen, die 
etwas gerundeter erscheinen, und konzentrische Anwachslamellen. Circummediterrangebiet und Portugal (Lissabon). Burdigalien und Sahélien.

Formen der Domeykoi-Gruppe mit flachen breiten Rippen in der Lk. Kl.

V. (Pecten) subbenedicta FonT. (16) T. 5, Fig. 1-2, S. 39. Die stark konvexe R. Kl. hat 12 gerundete Rippen, die breiter als die Zwischenräume, die Lk. Kl. 12 Rippen von rechteckigem Querschnitt, schmäler wie die Zwischenräume. Die konzentrische Verzierung der R.Kl. ist sehr schwach, die der Lk. deutlich. Die Berippung der R. Kl. entspricht vollkommen der V. Domeykoi PHIL. aus dem Lias und der V. cristatocostata SACCO (FUCHs'sche Abd.), nur sind die Rippen etwas mehr abgeplattet, mit flacherem Seitenabfall. Burdigalien, bes. für das Rhônebecken charakteristisch.

V. (Pecten) corsicanus DeP. (16) T. 4, Fig. 8-9, S. 37. Die R. Kl. ist stark gewölbt, mit 12 gerundeten Hauptrippen und 2-3 kleineren Flankenrippen. Es sind feine einfache Anwachslinien vorhanden. Die Lk. Kl. trägt 14 subquadratische, etwas gerundete Rippen, schmäler als die Zwischenräume. Die V. corsicana ist der $V$. subbenedicta außerordentlich ähnlich. Miozän von Korsika.

V. (Pecten) Benedicta LaM. (16) T. 4, Fig. 1-5, S. 33 ist von der V. subbenedicta FonT. hauptsächlich durch die schwächer gewölbte R. Kl. und die, wenigstens in der Nähe des Unterrandes, mehr gerundeten Rippen unterschieden. Ob. Miozän, am häufigsten Pliozän. Circummediterrangebiet and Rhônebecken.

\section{Vola-Formen mit glatter Lk. Kl.}

Die Formen mit breiten, aber flachen Rippen der Lk. Kl., sowohl in dem Alata-Stamm s. str. wie im Domeykoi-Stamm, sind durch Übergänge mit Formen verbunden, die nichts weiter darstellen wie Varietäten der normalen Formen mit extrem schwachen bezw. ganz verschwundenen Rippen. Nach DEPERET entsprechen sich:

\begin{tabular}{|c|c|c|}
\hline & Normale Rippen: & Abgeschwächte, bezw. keine Rippen: \\
\hline \multirow[b]{2}{*}{ Domeykoi-Gruppe } & V. Benedicta LAM. & V. Paulensis FoN. \\
\hline & V. subbenedicta Fon. & $\left\{\begin{array}{l}\text { V. subbenedicla Fox. var. Laevis DEP. } \\
\text { V. revoluta MICHELOTII }\end{array}\right.$ \\
\hline Alata-Gruppe s.str. & V. Josslingi SMIтH & V. Josslingi SMITH var. Laevis Cotт. \\
\hline
\end{tabular}


Jacobaea- und Aduncus-Gruppe mit sekundär komplizierter

\section{Berippung}

Im jüngsten Tertiär bis zur Gegenwart tritt eine sehr bekannte und noch lebend häufige Form auf, V. (Pecten) Jacobaea LINNĖ (16) T. 8, Fig. 1, S. 58, die stark sekundär geteilte Rippen in der R. Kl. besitzt. Die mäßig stark gewölbte R. Kl. mit mäßig eingekrümmtem Wirbel trägt 12 quadratische Hauptrippen mit je 8 Sekundärrippen, zu jeder Séite 2-3 kleinere Seitenrippen. Rippen breiter als die ungeteilten Zwischenräume. Bei jungen Individuen sind die Rippen noch einfach. Die Lk. Kl. ist in der Jugend deutlich konkav, im Alter mehr oder weniger eben, nur noch in der Wirbelgegend konkav, bei einem großen Exemplar ist sie sogar schon schwach konvex. Sie trägt 12 Rippen von rechteckigem Querschnitt, schmäler als die Zwischenräume und beide einfach. Die Zwischenräume wie auch die Rippen tragen in beiden Klappen konzentrische, ganz feine, lamellöse Anwachsverzierung. Denken wir uns die Sekundärberippung fort, so resultiert eine Form, die in der Berippung der R. Kl. vollkommen mit $V$. alata übereinstimmt. Wir finden die gleichen leistenförmigen, stark hervortretenden Rippen, die senkrecht zu den Zwischenräumen abfallen. Rippen der Lk. Kl. sind nicht so scharf wie bei V. alata, sondern auf dem Rücken mehr flach. Circummediterrangebiet; Pliozän - Rezent. V. Jacobaea ohne Sekundärrippen würde also eine Form ergeben, die im wesentlichen mit d en Vertretern des Alata-Stammes s. str. übereinstimmt, die in der Lk. Kl. breite Rippen aufweisen, also etwa V. (Pecten) Josslingi SмiтH., V. (Pecten) corsicana DËP. (vergl. S. 298-299). Die typische, schwach lamellenartige Querverzierung finden wir auch bereits bei $\nabla$. alata. Die R. Kl. ist allerdings weniger stark konvex und der Wirbel nicht so stark eingekrümmt. Auf diesen Unterschied werde ich später zurückkommen. Nach PHWIPPI (31) S. 113 ist der Übergang zwischen glatten und sekundär berippten Formen durch zahleiche Zwischenformen hergestellt.

Fine solche Übergangsform ist $V$. (Pecten) Grayi MrcH. (16) T. 8, Fig. 2, S.60. Sie ist von $\nabla$. Jacobaea durch die stärker gekrümmte konvexe Klappe, den stärker eingekrümmten Wirbel und ihre geringe GröBe unterschieden, Merkmale, die sie den Tertiärformen ohne Sekundärberippung näherbringen. Die sekundäre Teilung der Rippen und die übrigen Merkmale sind bereits wie bei $V$. Jacobaea. Auch DEPERET sieht in $V$. Grayi den direkten Vorläufer von $V$. Jacobaea, womit das zeitliche Auftreten im Miozän übereinstimmt. Bezeichnend ist, daß man $V$. Grayi auch als $V$. Praejacobaea BRIVES bezeichnet hat. Mediterranregion; Miozän. Weiterhin 
tritt im Tertiär noch eine Vota-Gruppe mit sekundärer Berippung auf, die aber von V. Jacobaea ganz verschieden ist. Die sekundären Bildungen sind sehr primitiv, weisen aber schon durch das Auftreten von Zwischenrippen mehr nach $V$. Bodenbenderi. Es ist das die Gruppe des P. aduncus EICHWALD (16) S. 49-57. In der Regel hat nur eine Klappe, und zwar die linke, Sekundärrippen, und das Verhalten von Zwischenrippen und Spaltrippen zueinander, von denen manchmal nur die einen entwickelt sind, der Grad der Entwicklung, machen einen noch viel primitiveren Eindruck wie bei $V$. Bodenbenderi. Bei V. Jacobaea ist auch nur ei e, aber die R. Kl., mit Sekundärrippen versehen. Zwischenrippen fehlen hier ganz, aber die Ausbildung macht keinen primitiven Eindruck, sondern den einer abgeschlossenen Entwicklung, die sich in einer anderen Richtung bewegt hat. Einige Typen dieser Aduncus-Gruppe mit sekundärer Rippenbildung sind:

\begin{tabular}{|c|c|c|c|}
\hline Namen & $\begin{array}{c}\text { Sekundärbildungen } \\
\text { der } \\
\text { rechten Klappe }\end{array}$ & $\begin{array}{c}\text { Sekundärbildnngen } \\
\text { der } \\
\text { linken Klappe }\end{array}$ & Vorkommen \\
\hline $\begin{array}{l}\text { 1. P. aduncus EICHW. } \\
\text { (DeP. T. } 6 \text {, Fig.5-6, } \\
\text { T. 7, Fig. 1, S. 49) }\end{array}$ & - & $\begin{array}{l}1 \text { Zwischenrippe, } \\
\text { Längsfurchen auf } \\
\text { den Hauptrippen. }\end{array}$ & $\begin{array}{l}\text { Wiener Becken, } \\
\text { Rhônebecken, } \\
\text { Atlant. Region. }\end{array}$ \\
\hline $\begin{array}{l}\text { 2. P. inflatus MrLlex. } \\
\text { (Dis. T.7, Fig.2-4, } \\
\text { S. 51) }\end{array}$ & - & $\begin{array}{l}1 \text { Sekundärrippe, } \\
2-3 \mathrm{Zwischen-} \\
\text { rippen. }\end{array}$ & $\begin{array}{l}\text { Zentral-Frankreich, } \\
\text { Helvétien. }\end{array}$ \\
\hline $\begin{array}{l}\text { 3. P. Dunceri MEYeR } \\
\text { (DEP. T.6, Fig.1-4, } \\
\text { S. 53) }\end{array}$ & - & 1 Zwischenrippe. & $\begin{array}{l}\text { Medit. Gebiet. Azo- } \\
\text { ren, Portugal, Hel- } \\
\text { vétien. }\end{array}$ \\
\hline $\begin{array}{l}\text { 4. P. Vigolensis SrM. } \\
\text { (DeP. T. } 7 \text {, Fig. } 5 \text {, } \\
\text { S. } 54 \text { ) }\end{array}$ & $\begin{array}{l}2-3 \text { Längsfurchen } \\
\text { auf den Haupt- } \\
\text { rippen }\end{array}$ & 1 Zwischenrippe. & $\begin{array}{l}\text { Medit. Gebiet. Hel- } \\
\text { vétien. }\end{array}$ \\
\hline
\end{tabular}

\section{Tertiäre Pectiniden mit Beziehungen zu den Vola-Formen des Alata-Stammes}

Die echten tertiären Pectiniden enthalten ebenfalls Formen, die unverkennbare Ähnlichkeit mit den Vertretern der liasischen Gruppe II aufweisen. Patinopecten (P.) caurinus Go0LD. (1), T. 38, Fig. 1, T. 39, Fig. 1-2, S. 101-103. Die .R. Kl. ist konvex, stärker als die Lk. Das vordere $\mathrm{Ohr}$ hat einen deutlichen Byssusausschnitt. Die R. Kl. trägt 20-25 quadratische, oben flache Rippen, die bei guter Erhaltung manchmal feine Radialstreifen erkennen lassen, Zwischenräume und 
Rippen sind gleich breit. Die Lk. flachere Kl. zeigt gerundete Rippen, die schmäler sind als die Zwischenräume. Beide Klappen haben feine konzentrische Anwachsstreifung. Pliozän - lebend. Kalifornien. Die außerordentliche Ähnlichkeit in der Berippung mit V. clata, auch was das Verhältnis von R. und Lk. Kl. zueinander angeht, ist nicht zu verkennen. Pecten bellus Conrad (1), T. 31, Fig. 1, S. 95-96. Die R. Kl. ist stärker gewölbt als die Lk., die in der Wirbelgegend sogar etwas konkav eingesenkt ist. Der Wirbel ist stark gekrümmt, Byssusausschnitt klein. Die R. Kl. hat 14-15 quadratische Rippen, schmäler als die Zwischenräume, mit flachem Rücken. Einige von ihnen zeigen gelegentlich 1-2 schwache Längslinien. Die Lk., schwächer gewölbte Kl. trägt 13-14 stark hervortretende, auf dem Rücken flache, zuweilen etwas bicarinate Rippen mit glatten, schräg abfallenden Flanken. Die Zwischenräıme sind breiter als die Rippen. Beide Klappen zeigen feinlamellöse, konzentrische Anwachsverzierung. Pliozän von Kalifornien. Die gleiche Art der Berippung in der Lk. Kl. haben wir schon bei echten Tertiär-Volen kennen gelernt, welche auch keine scharfen Rippen in der Lk. Kl. besitzen, sondern solche mit flachem Rücken (vergl. S. 298). Hiervon, sowie von der Bikonvexität und dem Byssusausschnitt abgesehen, kann ich keinen wesentlichen Unterschied gegenüber der $V$. alata aus dem Lias angeben.

Bei V. Jacobaea LrNNÉ habe ich schon darauf hingewiesen, daß die Lk. Kl. bei älteren Exemplaren flach, ja sogar schwach konvex werden kann. Formen, die zwei deutliche konvexe Klappen besitzen und einen Byssusausschnitt, also echte Pectiniden, mit den so charakteristisch verzierten Rippen von V. Jacobaea sind ebenfalls bekannt wie P. Stearnsii Datc (1), T. 32, Fig. 1, S. 100 aus dem Pliozän und P. Diegensis DALL (1), T. 51, Fig. 1, S. 127 aus dem Pliozän bis jetzt in Kalifornien. Bei beiden ist die Rippenzahl allerdings erheblich höher als bei $\nabla$. Jacobaea, über 20 Rippen. In der R. Kl. besitzen die Rippen einen rechteckigen Querschnitt, breiter wie die Zwischenräume, und sind durch Sekundärrippen zerteilt. In der Lk. Kl. sind die Rippen bei $V$. Stearnsii viel schmäler, bei $V$. Diegensis ungefähr gerade so breit wie die Zwischenräume. Zwischenräume und Rippen der Lk. Kl. sind einfach. Beide Arten zeigen auf beiden Klappen eine fein-lamellöse, konzentrische Anwachsverzierung. Die R. Kl. ist bei beiden konvex, die Lk. in der Wirbelgegend konkav. Sonst ist die Lk. Kl. bei $V$. Stearnsii konkav-eben, bei $V$. Diegensis flach oder auch mäßig konvex. Alle diese Merkmale stimmen mit der V. Jacobaea ïberein. Der einzige Unterschied ist die größere Rippenzahl, der Byssus- 
ausschnitt und die schwach-konvexe Lk. Kl. Letzterer Unterschied ist aber auch nicht so durchgreifend, da, wie wir wisssn, bei $V$. Jacobaea die Lk. Kl. im Alter schon schwach-konvex, bei den kalifornischen Formen aber eben bezw. schwach-konkav werden kann. Doch ist immerhin bei $V$. Jacobaea die konkave, bei V. Diegensis und V. Stearnsii die konvexe bis ebene Lk. Kl. Normalzustand. Was den Byssusausschnitt anbelangt, so scheint er mir bei der großen $V$. Jacobaea auch schon vielleicht schwach angedeutet zu sein.

Zum Schluß noch einige allgemeine Beziehungen zwischen den Lias-Formen und den jüngeren Vertretern. Vergleichen wir das auf Seite 282 beschriebene und Fig. 7 abgebildete Schloß von $V$. alata mit dem Schloß der V. Jacobaea oder den Schloßabbildungen tertiärer Formen (23), T. 14 u. 15, so finden wir eine absolute Übereinstimmung. Der einzige Unterschied besteht darin, daß bei den jüngeren Formen an Stelle einer Rinne mit senkrechten Zahnplättchen mehrere vorhanden sind, durch untereinander und mit dem Schloßrand subparallele Leisten getrennt. Ich kann hierin aber keinen prinzipiellen Unterschied erblicken, sondern nur eine im Verlauf der Entwicklung (Lias-Tertiär!) erworbene Komplikation. Sodann sei auf die vollkommene Übereinstimmung der Innenansicht der R. Kl. bei $V$. alata, den tertiären und lebenden Formen hingewiesen. Ganz die gleiche Innenansicht wie bei V. alata (vergl. Fig. 6) zeigen die FucHs'schen Abbildungen von Tertiär-Formen: Die Rippen sind quadratisch, mit eingesenktem Rücken und etwas aufgewulsteten Rändern. Die Zwischenräume senken sich plötzlich am unteren Schalenrand stark ein und verbreitern sich, während die.Rippen sich verschmäleru.

Mehrere Skulptureinzelheiten, die ich bei $V$. alata beschrieben habe, habe ich bei den jüngeren Vola-Formen nicht wiederfinden können. Das gilt zunächst von den feinen Wülstchen, die sich bei $V$. alata auf dem Steinkern beider Klappen dort finden, wo das Ohr an die Schale stößt. (Abd. 7 bei $x$ ). Ferner ist mir das feine System schräg verlaufender Linien, das sich bei der Liasform auf dem Raum zwischen der letzten Flankenrippe und dem $\mathrm{Ohr}$ befindet, bei jüngeren $\nabla$. nicht bekannt (wohl bei echten Pectiniden), vergl. S. 308. Das gleiche gilt von den Furchen auf den Flanken der Rippen der Lk. Kl. und dem feinen, linienartigen Wulst, der sich auf dem untersten Teil des Seitenabfalls der Rippen der R. Kl. findet; eine solche Skulptur ist aber auch bei echten Pectiniden nicht bekannt ist. Es sei aber nochmals darauf hingewiesen, daß sich diese letztgenannte Skulptur der R. Kl. auch bei V. alata nur im Jugendstadium deutlich findet, im Alter aber verwischt. 
Diese letztgenannten Differenzen können aber an der feststehenden Tatsache einer weitgehenden Übereinstimmung zwischen Volen des Tertiär und des Lias in den wesentlichsten Merkmalen nichts ändern.

\section{Vola aus der Kreide}

Aus der Kreide sind Vola-Arten zahlreich bekannt. Sie sind, wie auch PHШLPPI hervorhebt, von den liasischen wie tertiären Volen im allg. so stark verschieden, daß an einen direkten Zusammengang ihrerseits mit den bekannten älteren und jüngeren Formèn nicht zu denken ist. Es sind das die Vertreter der Atava-Gruppe, nach $\nabla$. (Pecten) atava Röm. (34), T. 18, Fig. 21, (35) S. 54. Hierhin gehören weiter V. quinquecostata Sow. (19), T. 144, Fig. 1-5, longicauda D'ORB. (19), T. 445, Fig. 9-14, quadricostata Sow. (19), T. 447, Fig. 1-7 usw. Das Typische für alle diese Arten ist das Auftreten von Rippen verschiedener Größenordnungen. Es treten in der Regel wenige, etwa 4-6 Rippen auf, die durch Größe alle anderen übertreffen, und eine größere Anzahl kleiner Rippen, die untereinander auch wieder verschieden sein können. Etwas Ähnliches ist bis jetzt weder aus dem Jura noch aus Tertiär und Gegenwart bekannt. Außer diesen Vola-Formen des Atava-Stammes mit ungleichen Rippen, finden sich aber, was PHIIPPI unbekannt war, in der Kreide auch noch zwei andere Typen: der eine weist eine vollkommen gleichmäßige Berippung auf und erinnert hierin, wie auch in vielem anderen, an $V$. alata, der andere zeigt zwar schon Differenzen in der Rippenstärke, die aber noch außerordentlich schwach sind, jedenfalls von dem echten Atava-Typus himmelweit entfernt. Es erscheint mir die Entstehung wenigstens eines Teiles der extrem ungleich stark berippten Formen aus gleichmäßig stark beripptén, wie $V$. alata, aus diesem Grunde recht wahrscheinlich, wohingegen andere der ungleich berippten Kreideformen wohl mit schon ungleich berippten palaeozoischen Vorfahren (Pterinaeen) in Verbindung zu bringen sind.

a) Kreide-Volen mit einfacher, gleich starker Berippung der ganzen Klappe

Aus der Kreide Vorderasiens ist eine Vola bekannt geworden, Vola Blankenhorni BRorr. (6), T. 2, Fig. 7, S. 15, die nur einfache Rippen trägt und in jeder Beziehung der V. alata so nahesteht, daß ich zwischen beiden keinen wesentlichen Unterschied entdecken kann. Die sehr klare Abbildung des Steinkerns stimmt mit meinem abgebildeten Alata-Kern 
vorzüglich überein. Die R. Kl. trägt 12 , schon in der Wirbelgegend sehr starke, leistenförmige Rippen mit senkrechtem Flankenabfall, welche nahe dem Wirbel schmäler, am Unterrand aber breiter sind als die Zwischenräume. Letzteres stimmt mit der V. alata vollkommen überein. Bei dieser sollen nach MöRICKE in der Regel 14 Rippen vorhanden sein, es können aber auch mehr, bis 16, und weniger vorkommen, wie bei meinen Exemplaren, die zum Teil nur 13 Rippen zeigen. Die Rippen der Lk. Kl. alternieren, genau wie bei V. alata, mit denen der R. Kl. und sind schmäler als diese. Sie würden also mit der Berippung der Lk. Kl. bei den erwachsenen Alata-Formen übereinstimmen, bei denen, wie S. 279 hervorgehoben, die scharfen Rippen der jungen Formen breiteren und flacheren Rippen Platz machen. Der V. Blankenhorni außerordentlich nahe steht, wie BroIII hervorhebt, $V$. decemcostata D'ORB. (19), T. 449, die sich dadurch von der asiatischen Art unterscheidet, „da $\beta$ sich hier je 2 Sekundärrippen zwischen die primären einschalten“. Vorkommen: Senon von Frankreich. Eine andere Kreideform mit vollkommen gleichmäßiger Berippung ist $V$. aequicostata LAM. (non D'ORB., T. 445 mit nicht ganz gleichmäßiger Berippung, wie auch CHOEFAT hervorhebt) ${ }^{1}$ ). Die Rippen der R. wie Lk. Kl. sind je untereinander vollkommen gleich, die R. Kl. mit breiteren Rippen als die Zwischenräume versehen, während auf der Lk. Kl. das Umgekehrte der Fall ist. Auf der R. Kl. zeigen die Rippen einen steilen, senkrechten Abfall zu den Zwischenränmen, vor allem in der Wirbelnähe, während sich das nach dem Schalenunterrand hin mehr verwischt. Der Rücken der Rippen ist breit gerundet oder, wenn abgewittert, abgeflacht. Das Verhältnis der Berippung auf den beiden Klappen zueinander und der Berippungstypus auf der R. Kl. sind also der gleiche wie bei den Liasformen. Form und Wölbungșverhältnisse, sowie die Wirbeleinkrümmung sind ebenfalls recht ähnlich. Der Hauptunterschied liegt in der größeren Rippenzahl und der damit zusammenhängenden, viel engeren Stellung der Rippen, wobei allerdings in Betracht zu ziehen ist, daB auch bei $V$. aequicostata nach SCHLAGINTWEIT die Rippenzahl erheblich schwankt: 25-30 Rippen bei Stiùcken aus Texas und Europa, 19-20 bei solchen aus Peru. Die beiden hier beschriebenen Kreide-Volen sind demnach den Liasformen im weitgehendsten MaBe ähnlich. Ein wesentlicher, charakteristischer Unterschied zwischen ihnen und ihren jurassischen Vorläufern existiert nicht.

1) V. aequicostata LAM. (non D'ORB.) (14), T. 3, Fig. 10, S. 152, dort weitere Literaturangabe. (39), S. 121. Bei SchLaginTwEIT ist wohl durch einen Druckfehler bei den Klappen rechts und links vertanscht. 
b) Kreide-Volen mit ganz geringen Unterschieden in der Stärke der Berippung

V. (Pecten) texanus Röm. (35̃), T. 8, Fig. 3, S. 65. Das RöMeR'sche Original zeigt eine stark gewölbte R. Kl. "mit 15-17 fast gleich großen, auf der Oberseite ebenen, glatten, ausstrahlenden Rippen bedeckt, welche durch glatte, kaum die halbe Breite der Rippen habende Furchen getrennt sind“. Die andere Klappe „ist eben und mit ganz flachen, ungleich ausstrahlenden Rippen bedeckt, deren ebene Zwischenräume den Rippen selbst an Breite gleichkommen". Die Berippung erinnert also zweifellos einerseits an $V$. alata, andererseits zeigen die Rippen aber doch schon die Neigung ungleich zu werden, wenn auch noch nicht in dem Maße, wie bei der Atava-Gruppe. „Bei der texanischen Art sind die Rippen von fast gleicher Stärke, und wenn ein Unterschied überhaupt bemerkbar ist, so ist jede dritte Rippe etwas stärker wie die zwischenliegenden." Ähnliche Verhältnisse zeigen noch manche anderen Formen, so z. B. die aequicostata D'ORB. (non LAM.). Auch hier ist die Ungleichheit der Rippen erst ganz schwach entwickelt. Jede fünfte Rippe ist auf der R. Kl. etwas stärker wie die zwischenliegenden. Ähnliches habe ich auch an Steinkernen beobachtet, die als $V$.quiadricostata und $V$.quinquecostata bezeichnet waren, aber von den beträchtlichen Unterschieden in der Stärke der einzelnen Rippen, wie sie D'ORBIGNY zeichnet, nichts erkennen ließen. Die Formen dieser Gruppe zeigen also bereits geringe Unterschiede in der Stärke der Rippen, die aber noch so schwach sind, daß sie hierin, wie auch in mancher anderen Beziehung, den Liasformen mindestens gerade so nahe stehen, wie den typischen Vertretern der Atava-Gruppe, die mit ihren oben skizzierten Merkmalen als dritte Gruppe von Kreide-Volen den zwei beschriebenen zuzugesellen ist. Es hat gewissermaßen den Anschein; als wenn bei Vola texana und ähnlichen Formen der Typus der Berippung der Atava-Gruppe erst (oder auch schon) ganz schwach angedeutet ist. Eine stärkere Ausbildung, eine fortschreitende Spezialisierung in der hier nur undeutlich, zaghaft angedeuteten Richtung $\mathrm{muß}$ zu Berippungstypen führen, wie sie in der Atava-Gruppe vorliegen.

\section{Liasische Pectiniden mit Beziehungen zu Lias-Volen}

Pecten Pradoanus VERn. et CoLL. (S. 289-90) ist von MÖRICKE zu Unrecht als Jugendform der $V$. alata angesehen worden. Ganz abgesehen von der völlig abweichenden Berippung, hat auch die ganz junge $V$. alata 
bereits die typisch konkav-konvexe Schale, während Pect. Pradoanus bikonvex bis plankonvex ist. Rein systematisch haben die beiden nichts miteinander zu tun, was natürlich, unter Berücksichtigung der rein morphologischen Grundlage, auf der unsere heutige Systematik aufgebaut ist, einen unter Umständen sehr engen phylogenetischen $\mathrm{Zu}-$ sammenhang keineswegs ausschließt. Die beiden Autoren der Spezies haben schon auf die große Ähnlichkeit mit der $V$. alata hingewiesen und, wie mir scheint, mit Recht. Die geringere Wölbung der Lk. Kl. ein Analogon zu den Wölbungsverhältnissen bei $V$. alata -, die Innenansicht, die starke Einkrümmung des Wirbels der R. Kl., der Bau des Schlosses, das System schräger Linien auf den Schalenflanken: alles ist bei beiden absolut gleich, nur daß sich bei $P$. Pradoanus diese letztere Skulptur auch noch auf die Ohren ausdehnt. Ein erheblicher Unterschied liegt in den breitrïckigen, rechteckigen Rippen der V. alata und den scharfdreieckigen des P. Pradoanus, doch kennen wir in der Gruppe der V. Domeykoi PHIL. (vergl. S. 291 u. 296) anch Lias-Volen mit schmalrückig-gerundeten, wenn auch noch nicht dreieckig-zugeschärften Rippen, die ihrerseits wieder mit den typischen Formen der Alata durch Übergänge verbunden sind. Übereinstimmend findet sich in beiden Fällen der merkwürdige, ganz feine Wulst auf dem unteren Teile des Abfalles der Rippenflanken und die starke Querverzierung der Rippen wie der Zwischenräume. Auch die Rippenzahl paßt ganz gut (14 bezw. 12).

Eine Form, die in sehr engen Beziehungen zu dem P. Pradoanus steht, ist $P$. ambongoensis THEV. aus dem Lias von Madagaskar (42), T. 4, Fig. 2-3, S. 24, „intéressant par sa charactère mixte entre les Chlamys aequivalves ou subaequivalves, souvent fixés par un byssus et les Volas aequilatérales, inaequivalves, sans ouverture d'une passage pour un byssuss". Die Schale trägt fast die gleiche Zahl scharf dreieckiger Rippen wie P. Pradoanus (12) und weist die gleichen Anwachsstreifen auf. Die Lk. Kl. ist in der Jugend (bis $15 \mathrm{~mm}$ ) konkav und zeigt von da an das Bestreben, durch Annahme der Konvexität die Gleichklappigkeit des echten Pecten zu erwerben. Die Übereinstimmung mit $P$. Pradoanus sowohl in dieser Beziehung, wie anch in bezug auf Berippung ist vollkommen. Die Stacheln, die sich auf der R. Kl., und zwar besonders auf den Flankenrippen finden, erscheinen mir als ein besonderes Speziesmerkmal, das für die Zugehörigkeit zu der ganzen Gruppe des P. Pradoanus indifferent ist. Eine andere Form, die $P$. Pradoanus und besonders $P$. ambongoensis sehr nahe steht, ist der P. Euthymei DUMORT. (21), Bd. 1, T. 10, Fig. 8-10, S. 64 aus dem unteren Lias des Rhonebeckens. Wir finden die gleichen, scharfen, 
dachfirstartigen Rippen wieder in nur wenig größerer Zahl (16), und die gleichen Anwachsstreifen. Eine außerordentliche Ähnlichkeit erhält die Spezies mit $P$. ambongoensis dadurch, daß auch hier jederseits die drei Flankenrippen kleine Stacheln tragen, die sich allerdings hier auf beiden Klappen finden. Eine sehr merkwürdige Übereinstimmung nit $P$. Pradoanus besteht in dem Strichsystem, das sich auf dem Raum zwischen der letzten Flankenrippe und dem Schalrand vorfindet (kommt auf der Fig. 11a nicht zum Ausdruck, stimmt aber auf der DUMORTLER'schen Abbildung vollkommen mit dem $P$. Pradoanus überein). Der gemeinsame Unterschied gegenüber $P$. Pradoanus und $P$. ambongoensis liegt, wenn wir die unwesentlichen Merkmale der Spezies, wie z. B. die Stachelbildung, ausschalten, in der hier bereits vollkommen erreichten Gleichklappigkeit und dem Byssusausschnitt. (Über die Bedeutung dieser Merkmale vergl. S. 316.) Eine andere Spezies, die hier vielleicht zu nennen ist, ist $P$. Thiollierei MarTin (21), Bd. 1, T. 10, Fig.4-7, S. 62, aus dem unteren Rhone-Lias. Die Berippung ist die gleiche wie bei P. Pradoanus, aber vielleicht sind die Rippen nicht ganz so scharf und zahlreich. Die Schale ist gleichklappig, mit Byssusausschnitt. Auf dem Schalenflankenteil ist dieselbe Skulptur wie bei P. Pradoanus und $V$. alata. Über diese merkwürdige Verzierung, die ich nur noch bei den südamerikanischen Vola-Formen beobachtet habe und sonst nirgends kenne, sagt Domorter: "Ce genre d'ornement paraît être commun à tous les Pectens de ce niveau, quelque disparat que soit la forme générale et les détails des côtes" Pecten Veyrasensis DuM. (21), Bd. 1, T. 24, Fig. 19, S. 163 ist ein typischer $P$. Pradoanus und nach Zahl und Form der Rippen absolut ident. Unterschiede sind der Byssus und vermntlich die Gleichklappigkeit. Da leider über letzteren Punkt in der Beschreibung nichts verlautet, so nehme ich solche als das Normale an. Die charakteristische Verzierung des Schalenflankenteils ist wie bei $P$. Pradoanus und den anderen genannten Formen des unteren Rhone-Lias vorhanden.

Dem $P$. Pradoanus ist ferner sehr ähnlich der $P$. cardioides Prw. (vergl. S. 290). Er hat dieselben, scharfen, dreieckigen Rippen in nahezu der gleichen Zahl (13-15). Die R. Kl. ist stark gewölbt, mit stark gebogenem Wirbel, und das Ohr hat (vergl. die Abbildung bei PHسIPPI) dieselben schiefen Rippen wie bei P. Pradoanus. Die gleiche Übereinstimmung herrscht im Schloßbau. Der einzige Unterschied ist, von der Größe abgesehen, die starke Wölbung der Lk. Kl. Das ist aber in meinen Angen kein prinzipieller Unterschied, sondern nur die intensivere Ausbildung eines bei $P$. Pradoanus schon angedenteten, aber noch nicht so stark entwickelten und ausgebildeten Merkmals, nämlich der, wenn ich 
so sagen kann, Pecten-Natur der Lk. Kl.; während bei P. Pradoanus die Lk. Kl. noch schwächer gewölbt ist oder eben werden kann, ist sie bei $P$. cardioides immer stark konvex. Es liegt meiner Meinung nach auch hier das typische Endglied einer Pectenreihe vor, die aus Vola entstanden ist, und in der P. Pradoanus eine Art Bindeglied zwischen dem Vola- und Pecten-Stadium bildet. P. cardioides repräsentiert hier das gleiche Entwicklungsstadium wie die zitierten bikonvexen Formen des Rhonebeckens, die aber, entsprechend ihren Größendimensionen, wohl der Spezies P. Pradoanus speziell näherstehen, wie $P$. cardioidcs. Die gleiche Stellung wie $P$. cardioides PHIL. haben P.lycorrhynchus PHIL. und P. uncus PHש. (32), T. 17, Fig. 1 u. 3, S. 29 u. 30, die sich beide, wie die Abbildung und Beschreibung PHILIPpI's zeigen, von $P$. Cardioides nur unwesentlich unterscheiden.

Eine andere Form von dem Typus der Pradoanus-Gruppe ist aus der patagonischen Kreide bekannt als P. octoplicatus STANT. (38), T. 4, Fig. 2-3, S. 14, wie der Name sagt mit 8 Rippen auf der stark gewölbten Klappe, also erheblich weniger wie bei $P$. Pradoanus. Sie sind dreieckig, etwa ebenso breit wie die Zwischenräume, manchmal mit undeutlichen, radialen Längsstreifen und stets von sehr scharfen, konzentrischen Anwachsstreifen gekreuzt. Der Zeichnung nach zu urteilen, ist auf den Rippen derselbe linienfeine Wulst im unteren Teile der Rippenflanken vorhanden, wie bei P. Pradoanus. Der Wirbel ist stark eingekrümmt, ein Byssusausschnitt vorhanden. Die Lk. Kl. ist schwächer konvex, die Rippen treten nicht so stark hervor, sind nicht so scharfdreieckig, ihre radialen Linien und konzentrischen Anwachsstreifen undeutlicher als auf der R. Kl. Der Typus der Berippung, das Verhalten der Wölbung und Berippung in den beiden Klappen zueinander, die konzentrischen Anwachsstreifen, alles das stimmt mit P. Pradoanus aufs beste überein. VERNEUIL et COLLOMB heben in ihrer Beschreibung besonders hervor, daß auf der $\mathrm{Lk}$. Kl. bei P. Pradoanus die Rippen nicht so stark hervortreten, was ich allerdings bei meinen Exemplar nicht beobachten konnte. Andererseits ist bei $P$. octoplicatus die Zahl der Rippen eine andere, und auch die eigenartige Verzierung der Ohren und Flanken fehlt der patagonischen Form, dazu ist ein deutlicher Byssusausschnitt vorhanden. Ich betrachte daher diese Kreidespezies als ihrem allg. Habitus nach als zur Pradoanus-Gruppe gehörend, möchte aber betonen, daß sie gerade zur Spezies P. Pradoanus nicht in besonders enger Beziehung $\mathrm{zu}$ stehen scheint. 


\section{E. Zusammenfassung}

Die Lias-Volen lassen sich in 2 Formenkreise teilen, solche mit

I. ungeteilten Rippen und Zwischenräumen, stark gewölbten Schalen und stark eingekrümmtem Wirbel und solche mit

II. weniger stark gewölbter Schale, weniger stark eingekrümmtem Wirbel, sekundär geteilten Rippen und Zwischenrippen in den Zwischenräumen, bezw. solche Formen, die eine dahingehende Tendenz zeigen, ohne daß diese Sekundärbildungen gleichsam vollendet und konsequent durchgeführt zu sein brauchen.

Beiden Formenkreisen begegnen wir im Tertiär, vom Oligozän bis zum jüngsten Tertiär bezw. bis jetzt wieder, und zwar sind sie durch Arten vertreten, die $\mathrm{zu}$ den liasischen sehr enge Beziehungen aufweisen: der Alata-Stamm im weitesten Sinne durch V. Jacobaea, die sich allerdings in jüngerer Zeit sekundäre Teilung der Hauptrippen zugelegt hat, der Bodenbenderi-Stamm durch V. grandis und V.Vasseli im jüngsten Pliozän. Unter den tertiären und lebenden Pectiniden mit 2 gewölbten Klappen finden sich gleichfalls Arten vom Berippungstypus beider Formenkreise, mit starken Anklängen an die Lias-Volen. Innerhalb des I. Formenkreises kann man zur Liaszeit 2 Gruppen absondern: A. Die Rippen der gewölbten R. Kl. sind auf dem Rücken oben breit, vorwiegend flach, mit mehr oder weniger steilem Abfall zu den Zwischenräumen, B. die Rippen der R. gewölbten Kl. sind auf dem Rücken oben schmal, gerundet, die Flanken fallen mehr oder weniger flach zu den Zwischenräumen $\mathrm{ab}$, so $\mathrm{da} \beta$ sie nach oben dachfirstartig konvergieren, ohne aber eine scharfe Kante zu bilden. Diese beiden Gruppen finden sich ebenfalls unter den Tertiär-Volen (bis zur Jetztzéit?) wieder, der Alata-Stamm s. str. zweifelsohne noch rezent in $\nabla$. Jacobaea mit später erworbener Sekundärteilung der Rippen. Die tertiären Gruppen enthalten Spezies, die große Ähnlichkeit mit den liasischen Ausgangsarten besitzen, wie im Vorhergehenden für einzelne Spezies nachgewiesen. Bei einem Teil von Tertiär-Formen - sowohl der Gruppe A wie B - finden wir in der Lk. Kl. scharfe, dreieckige Rippen, genau wie bei den Liasformen, bei einem anderen Teil, der sich ebenfalls auf beide Gruppen verteilt, sind die Rippen der Lk. Kl. flach bis eben, von wechselnder Breite, die indessen in der Regel hinter der der Zwischenräume zurückbleibt. Ein weiterer Formenkreis im Tertiär zeigt einen auffälligen Rückgang in der Berippung, der bis zum völligen Ver- 
schwinden derselben führen kann. Diese Arten sind durch deutliche Übergänge mit den normal berippten Formen beider Kreise verbunden. Ferner zeigt eine Tertiär-Gruppe, welche durch Übergänge mit den normalen Formen der Gruppe A verbunden ist, eine sekundäre Teilung der Rippen der R. Kl., die aber nichts mit der beschriebenen, sehr viel älteren Bildung sekundärer Rippen in der Bodenbenderi-Gruppe zu tun hat. Unter den tertiären echten Pectiniden finden sich die beiden Gruppen A und B ebenfalls wieder und zwar mit größter Ähnlichkeit zu den Liasformen.

Unter den Kreideformen gibt es auch einzelne echte Vertreter des gleichmäßig berippten Liasstammes. Die stark ungleich berippten Formen, das Gros der Kreide-Volen, sind vielleicht auch z. T. durch nur schwach differenzierte Typen mit den gleichmäßig berippten Liastypen verbunden, so daß die Entstehung mancher (nicht aller!!!) ungleich stark berippter Kreideformen aus gleichmäßig berippten Vorfahren wahrscheinlich wird.

\section{F. Folgerungen}

In seiner Stammesgeschichte der Pectiniden (31) ist PHILIPPI bezüglich der Phylogenie von Vola zu Resultaten gekommen, die ich auf Grund meiner Beobachtungen an Lias- und Tertiär-Formen nicht bestätigen kann. Er ist der Ansicht, „daB Janiren aus Pectiniden iterativ zur Lias-,.Kreide- und Tertiärzeit entstanden sind. Zur Frage, ob Vola überhaupt aus Pecten entstanden ist, vergl. S. 316. Mit P. aequivalvis Sow. und priscus ScHOLTH. haben die Liasformen jedenfalls nichts zu tun. Vergleichen wir zunächst $P$. aequivalvis Sow. $\left.{ }^{1}\right)$ mit $V$. alata, so hat ersterer eine viel größere Rippenzahl, etwa 20, und die Rippen sind im allg. gleichmäßig konvex gerundet und zeigen nichts von den stark hervortretenden, dick-leistenförmigen Rippen der $V$. alata. Thre Form scheint allerdings etwas zu variieren. Es gibt Exemplare, bei denen sie auf dem Rücken stärker abgeplattet sind, und solche, bei denen sie etwas zugespitzt sind. Von dem großen Unterschied der Berippung in den beiden Klappen, wie ihn $\nabla$. alata zeigt, ist nichts vorhanden. Die Schalen können verschieden stark gewölbt sein, haben aber niemals die außerordentlich starke Konvexität wie die R. Kl. der Liasform, auch ist der Wirbel niemals stark eingekrümmt. Von der eigenartigen Verzierung der Schalenflanken in Gestalt schief verlaufender Rippen ist ebenfalls bei $P$. aequivalvis Sow. keine Spur zu

1) (33) T. 23, Fig. 1, S. 183; (13) T. 32, Fig. 1, S. 212; (24) S. 89, Fig. 4, S. 23. 
entdecken. $V$. Bodenbenderi mit seinen kompliziert gebauten Rippen hat erst recht keine Spur Ähnlichkeit mit der zitierten Art. Ebensowenig wie $V$. alata mit $P$. aequivalvis, ebensowenig hat $P$. (nicht $V$. ) Pradoanus mit $P$. priscus ScHoLTH. zu tun. Es sind fast die gleichen Unterschiede wie zwischen $V$. alata und $P$. aequivalvis: die Rippenzahl bei $P$. priscus ist fast die doppelte $(20-24)$ wie bei $P$. Pradoanus. Der Wirbel ist nicht eingekrümmt, wie es BEHRENDSEN von $P$. Pradoanus angibt. Die Rippen sind niemals so typisch scharf und dreieckig, GoLDFuss gibt sogar auf der einen Klappe konvexe Rippen an, etwas breiter wie die Zwischenräume, auf der anderen Klappe "ein wenig zugeschärft". Die Zwischenräume der Rippen sind in beiden Schalen konvex und nicht scharf dreieckig. Von der eigenartigen Verzierung der Ohren, wie sie $P$. Pradoanus zeigt, ist keine Spur vorhanden, ebensowenig von der sehr typischen, feinen, lamellösen Anwachsstreifung.

Die tertiären Volen leitet PHسIPPI (wie er selbst sagt, fehlen Übergangsformen!!) von der Plebejus-Gruppe seines Aequipecten-Stammes ab, zu der er u. a. stellt:

P. plebejus LaM. (17) Bd. 2, S. 82; (26) Bd. 2, T. 39, Fig. 16, S. 916: Pariser Becken

P. piclus GoLDF. (24) T. 97, Fig. 4, S. 67

$P$ : decussatus MüNsT. (24) T. 94, Fig. 5, S. 65

P. lineatus GoLdF. (24) T. 94, Fig. 6, S. 59 Norddeutschland

Plebejus-

Gruppe

PhILIPPI

Ich habe diese Formen daraufhin durchgesehen und auch nicht die geringste Spur von Ähnlichkeit mit den Tertiär-Volen entdecken können, so daß ich einen derartigen Zusammenhang für ganz ausgeschlossen halte. Aus diesem Grunde, und vor allem auf Grund der ausführlich beschriebenen Beobachtungen komme ich

I. zu dem negativen Resultat, daß die von PHILIPPI versuchte Ableitung der Lias- und Tertiär-Volen unrichtig ist,

II. zu dem positiven Ergebnis, daß hier keine iterativen Bildungen vorliegen, sondern daß die Lias-Volen in phylogenetischem Zusammenhang mit manchen Kreideformen, und vor allem mit den tertiären und lebenden Vola-Arten stehen, in der Weise, daB sich mehrere, schon zur Jurazeit wohl unterscheidbare Formenkreise bis in die jüngste Zeit verfolgen lassen. 
Tertiär-Volen mit reduzierter Berippung, die ihrem ganzen Habitus nach z. T. zu der Alata-Gruppe s. str., z. T. zur Domeykoi-Gruppe gehören, sind in größerer Zahl bekannt; sie zeigen schwache oder auch überhaupt keine Rippen in der Lk. Kl. und sind mit normalberippten Tertiärformen durch Übergänge verbunden und zweifelsohne erst im Tertiär von diesen abgezweigt. Vielleicht trifft dasselbe für diejenigen tertiären Formen des Alata-Gruppe s. str. wie auch der Domeykoi-Gruppe zu, die an Stelle der scharfen Rippen der Lk. Kl. flache, breite aufweisen. Vielleicht können letztere Formen aber auch schon zur Liaszeit existiert haben, das kann ich an der Hand meines Materials nicht entscheiden. Ebenso ist es mir wahrscheinlich, daß die liasische Bodenbenderi-Gruppe von einfachen Alata-Formen abstammt. Die V. Bodenbenderi zeigt noch anf der R. KKl. durchaus unregelmäBige, auf den verschiedenen Rippen verschiedenartige Sekundärrippenbildung, es macht den Eindruck, als wenn wir den ersten, aber noch nicht vollkommenen Versuch einer einfachen Form vor uns hätten, Sekundärrippen auszubilden. Es würden dann die beiden Gruppen in präliasischer Zeit auf einen gemeinsamen Stamm vom Typus der Alata-Gruppe zurückgehen. Wann und wo diese Sonderung sich vollzogen hat, ist zurzeit noch nicht entscheidbar, sicher aber in präliasischer Zeit.

Unter den Tertiär-Volen mit Sekundärrippen erscheint die Aduncus-Gruppe mit ihren höchst primitiven sekundären Bildungen als eine zweite, jüngere Tendenz, aus einfacher Berippung kompliziertere entstehen zu lassen, als eine Umbildung, die sich in dem gleichen Sinne vollzieht wie bei der $V$. Bodenbenderi im Lias. Diese tertiäre Gruppe ist erst in tertiärer Zeit aus einfachen Formen entstanden. Es sind keine direkten Nachkommen von $V$. Bodenbenderi aus dem Lias, sondern es ist eine analoge, jüngere Parallelreihe. Der Vorgang der Sekundärrippenbildung scheint sich also zu verschiedenen Zeiten in verschiedenen Stämmen wiederholt zu haben, ohne daß aber von „iterativer Artbildung“ die Rede sein kann. Ich möchte noch hinzufügen, daß diese Art von Sekundärrippenbildung bei Vola immer in der Lk. Kl. begonnen zu haben scheint: bei $\nabla$. Bodenbenderi ist sie in der Lk. Kl. stärker entwickelt wie in der R. Kl., bei Tertiär-Formen fehlt sie z. T. noch in der R. Kl. V. (Pectcn) grandis Sow. aus dem Jungtertiär mit Sekundärrippen und Zwischenrippen ist als direkter Nachkomme des Bodenbenderi-Stammes aus dem Lias anzusehen. Der dritte tertiäre Formenkreis mit Sekundärberippung, V. Jacobaea LINN. (jungtertiär bis rezent), zeigt ganz andere Sekundärrippenbildung, die mit der Bodenbenderi-Gruppe, zu der schließ- 
lich im weitesten Sinne als junger Parallelast auch die Aduncus-Gruppe gehört, gar nichts zu tun hat. Sie ist in jungtertiärer Zeit aus der Alata-Gruppe s. str. hervorgegangen, wie schon PHuIPPI annimmt, und zwar wahrscheinlich aus den tertiären Formen mit breiten Rippen in der Lk. Kl. Hier sind uns auch die Übergangsformen bekannt. Unter den tertiären Janiren mit Sekundärberippung haben wir also drei phylogenetisch verschiedenwertige Abteilungen. Die Jacobaea-Gruppe bei DEPERET (16) S. 56 stellt bloß systematisch, nicht entwicklungsgeschichtlich eine Einheit dar.

Unter den Vola-Formen der Kreide findet sich zunächst im Gegensatz zu der PPIIIPPI'schen Behauptung der Alata-Stamm s.str. unzweifelhaft wieder (V. Blankenhorni BroIL., V. aequicostata LAM.), und zwar u. a. in einer Spezies (V. Blankenhorni), die sogar speziell der Spezies V. alata nahesteht. Des weiteren findet sich der Atava-Stamm mit seinen extrem ungleichen Rippen gewissermaßen als der entgegengesetzte Pol vom Neokom bis Senon. Eine Zwischenstellung, rein morphologisch betrachtet, nehmen die Formen ein (V. texana, V. aequivalvis), die noch fast gleichmäBig stark berippt sind, aber schon eine schwache Andeutung zur Anlage verschieden starker Rippen zeigen, welche in diesem Sinne weiter ausgebildet gedacht, zu Atava-Formen führen muß. Die gleichmäßig berippten Formen finden sich schon im Lias und zwar dort $\mathrm{nur}$ solche gleichmäßig stark berippten. In der Kreide dominieren bei weitem die stark ungleich berippten Typen, daneben finden sich aber auch noch Formen des Alata-Typus und solche mit ganz geringer Ungleichheit der Rippen. Auf Grund dieser Tatsachen bin ich der Ansicht, daB manche (aber sicher nicht alle, vergl. S. 304, 306 u. 311) Atava-Formen aus gleichmäBig stark berippten Alata-Typen entstanden sind, und sehe als $\mathrm{Zwischenglieder}$ die V.texana-aequicostata-Formen an. Es muß diese Umbildung in den verschiedenen Gruppen zu sehr verschiedenen Zeiten stattgefunden haben, jedoch nach dem jetzigen Stand unserer Kenntnisse erst postliasisch, da sich in der Kreide noch alle drei Typen nebeneinander finden, wenn auch quantitativ sehr ungleich. Bei diesen Erwägungen handelt es sich natürlich bloß um die mögliche phylogenetische Verknüpfung großer Formengruppen, die ich andeuten möchte. Über den Zusammenhang einzelner Spezies läßt sich bei der Spärlichkeit des Juramaterials nichts Sicheres sagen. Eine Atava-Form, die vielfach mit einer Alata-Form in Verbindung zu bringen wäre, könnte nach BROILI $V$. decemcostata D'ORB. und $V$. Blankenhorni BrolL. sein. Glaube ich über die Herkunft eines Teiles der Kreide-Volen einige feste Anhaltspunkte gewonnen zu haben, so muß ich das leider 
für die Frage, was aus ihnen in postcretacischer Zeit geworden ist, vollkommen verneinen.

Unter den tertiären und rezenten Pectiniden finden sich Formen, die mit der Alata- wie Bodenbenderi-Gruppe bezw. deren einzelnen Zweigen, und zwar schon mit den liasischen Vertretern, die größte Übereinstimmung aufweisen, so daß sie als ans echten Janiren hervorgegangen zu betrachten sind. Zwischen dem, ich möchte sagen, Janiraund Pecten-Stadium des Jacobaea-Zweiges kennen wir die Übergänge in der Tat, und auch das zeitliche Auftreten stimmt mit dieser Annahme überein. Bemerkenswert ist die noch immer geringere Konvexität der Lk. Kl. bei den jungtertiären und rezenten Pectiniden, die aus Janira entstanden sind.

Ebenso gibt es Lias-Pectiniden, wie den P. Pradoanus (S. 307), dessen weitgehende Übereinstimmung mit der $\nabla$. alata es wahrscheinlich macht, daß es auch alata-ähnliche Formen mit scharfen, dreieckigen Rippen wie $P$. Pradoanus gibt, die uns aber zurzeit noch nicht bekannt sind, und daß aus solchen Formen der P. Pradoanus durch Verlust der Konvexo-Konkavität und Annahme der Bikonvexität hervorgegangen ist. Ich sehe also im P. Pradoanus eine Vola, die den Pecten-Typus angenommen hat, genau wie wir das für gewisse Tertiär-Pectiniden oben kennen gelernt haben. Die Richtigkeit dieser Vermutung wird durch den dem P. Pradoanus sehr nahestehenden madagassischen $P$. ambongoensis bewiesen, der, in der Jugend selbst noch Vola, im Alter bikonvex, also echter Pecten ist, dessen Ontogenese demnach die Entwicklung Vola-Pecten zeigt. Es ist das keine Vermutung, sondern feststehende Tatsache. Durch die dem $P$. Pradoanus und $P$. ambongoensis so überaus ähnlichen Formen aus dem Rhonebecken (S. 308) wird weiterhin bewiesen, daB aus dem intermediären Durchgangsstadium des Pradoanus-Typus durch Weiterausbildung der Entwicklungsrichtung, die vom Vola: Stadium zum Pradoanus geführt hat, echte, gleichklappige Pectiniden entstanden sind. Wir hätten hier also einen zweiten Fall, in dem tatsächlich die Entwicklung von Pecten aus Vola - nicht umgekehrt - mit Zwischengliedern erwiesen ist. Diese Umformung muß in präliasischer Zeit vor sich gegangen sein, da der fertige Pecten schon im Lias vorliegt, was natürlich nicht ausschließt, daß die zugehörige Vola-Form daneben auch noch im Lias existiert hat und später vielleicht auch noch aufgefunden werden wird. Jedenfalls hat meine Erklärung den Vorzug, daß sie sich zwanglos in den Rahmen der allgemeinen Beziehungen zwischen Vola und Pecten (S. 307) einfügt, d. h. Pecten als 
die sekundäre und Vola als die primäre Form hinstellt und keine Formen miteinander in Verbindung bringt, wie P. priscus und P. Pradoanus, die nichts miteinander zu tun haben. Der Pect. octoplicatus (S. 309) aus der patagonischen Kreide stellt phylogenetisch ein dem PradoanusStadium gleichwertiges Element dar. Das Vola-Stadium speziell zu dieser Spezies ist zurzeit noch ebensowenig bekannt wie das zu $P$. Pradoanus gehörige. $\mathrm{Ob}$ der $P$. octoplicatus sich erst im Verlauf der Kreide oder bereits früher aus dem Vola-Stadium entwickelt hat und im letzteren Falle also einen Nachzügler einer älteren Form in der Kreide darstellt, ist auch noch nicht entscheidbar.

Die Janiren haben bei der Umbildung zu Pectiniden 1. die KonvexoKonkavität verloren, 2. einen Byssus-Ausschnitt erworben, d.h. sie sind von der freibeweglichen Lebensweise höchstwahrscheinlich zu der festsitzenden übergegangen ${ }^{1}$ ). Hiermit ist begreiflicherweise eine Abnahme der Konvexität der R. Kl. und der Wirbeleinkrümmung Hand in Hand gegangen. Wir kennen zwei sichere Beispiele mit Übergangsformen (vor allem die Ontogenie des $P$. ambongoensis) und mehrere sehr wahrscheinliche, zu denen aber noch die Zwischenformen im einzelnen fehlen, die mit positiver Sicherheit beweisen, daB im Tertiär bezw. präliasisch aus Janiren Pecten entstanden sind. PHسLPPI nimmt umgekehrt an, daß Janira von Pecten abstammt, bleibt aber hierfür den Beweis schuldig. Da beide Entwicklungsmöglichkeiten nebeneinander, also gewissermaßen nach Belieben bald Janira-Pecten, bald PectenJanira, unmöglich sind, und mehrere sichere Beispiele für die Entwicklung Janira-Pecten nachgewiesen sind, so behaupte ich, daß Janira überhaupt nicht aus Pecten entstanden ist, sondern daß das Umgekehrte der Fall ist ${ }^{1}$ ). Es ist das der dritte Punkt, in dem ich durch meine Untersuchungen zu dem Resultat gekommen bin, daß die PHILIPPI'schen Ansichten unzutreffend sind: aus konkav-konvexen Janiren sind bikonvexe Pecten-Formen entstanden, und die Lias-Janiren sind die Nachkommen palaeozoischer, zurzeit noch nicht näher bekannter, Zweischaler mit konkav-konvexen Klappen.

Wir haben also im Lias von Südamerika zwei Haupttypen von Vola mit mehreren Untergruppen, die vielleicht in präliasischer Zeit aus einem gemeinsamen Stamme entstanden

1) „Höchstwahrscheinlich“, da nach JACKsoNs Untersuchungen (27) S. 328 unten der Byssusausschnitt nicht nur dem Vorhandensein eines Byssus, sondern unter Umständen auch anderen Ursachen seine Entstehung verdanken kann. 
sind. Die Nachkommen eines Hauptstammes finden wir in der Kreide wieder. Die Nachkommen beider Hauptstämme, mit ihren Untergruppen, finden wir im Tertiär wieder und können sie bis zum jüngsten Tertiär bezw. der Gegenwart verfolgen. Den einen Stamm kennen wir also ununterbrochen vom Lias bis jüngsten Tertiär bezw. der Gegenwart, von dem zweiten Stamm sind die Kreideformen zurzeit noch nicht bekannt. Zu verschiedenen Zeiten haben sich in verschiedenen $\mathrm{Zweigen}$ der Vola-Stämme Umbildungen wiederholt, mit gleichzielender Tendenz, wenn ich einmal das Wort hier anwenden darf, aber keine iterative Artbildung. Aus den Janiren sind durch Verlust ihrer Konvexo-Konkavität und Annahme eines Byssusausschnittes, also einer festsitzenden Lebensweise, bereits präliasisch und im Tertiär bikonvexe Pecten geworden. Die umgekehrte Entwicklung hat niemals stattgefunden, und die liasischen Volen müssen die Nachkommen konvex-konkaver, palaeozoischer Vorfahren sein.

1) Selbstverständlich sind nicht alle Pectiniden aus Vola entstanden, sondern meine Behauptung gilt nur für einen kleinen Teil dieser außerordentlich umfangreichen Familie. 


\section{Literaturverzeichnis}

1. B. ARNold. The tertiary and quarternary Pectens of California. Prof. paper U. S. Geol. Surv. No. 47. Ser. C. Systematic Geology and Palaeontology. 1906.

2. E. Bayle et Coquand. Memoires sur les fossiles secondaires récueillis dans le Chili par M. Domeyko et sur les terrains auxquels ils appartiennent. M.S. G.F. Ser. 2, Bd. 4. 1851-52.

3. O. Behrendsen. Zur Geol. des 0.-Abhanges der argent. Cordillere. Z. D. G. G. 1891, Bd. 43, Teil 1.

4. M. Blankenhorn. Die Vola-Arten des ägyptisch. u. syrisch. Neogens. N. J. Bl. Bd. 17. 1903.

5. B. Brocchr. Conchiologia fossila subappennina Milano. 1843.

6. F. Broili. Geolog. u. palaeont. Resultate der Grotheschen Vorderasien-Exped. 1906-7.

7. G. Brons. Lethaea geognostica. Heidelberg 1852-56.

8. L. von BucH. Ueber die Jura-Formation auf der Erdoberfläche. Monatsber. Kgl. Akad. d. Wiss. Berlin 1852.

9. L. von Buch. Petrifications recueillis en Amériques par Humboldt et Degenhardt. Berlin, 1839.

10. C. Burckhardt. Beitr. z. Kenntnis des Jura und der Kreide der Cordillere. Palaeontographica, Bd. 50.

11. C. Burckmandt. Profiles Géol. transversaux de la Cordillère argentino-chilienne. Ann. del Museo de la Plata. Sećcion geolog. y mineralog. 1892-1900. Teil 2.

12. H. Burmeister und C. Gifbel. Die Verstein. von Juntas im Tale des Rio de Copiapó. Abh. naturf. Ges. Halle, Bd. 6. 1861.

13. F. Chaputs et Dewalque. Descript. des fossiles des terrains second. de la province de Luxembourg (mit Suppl.). Brüssel 1853, 1858.

14. P. Choffat. Recueil d'études palaeont. sur la faune crétacique du Portugal. 4. Ser. Lisbonne 1901/02.

15. D'Archiac. Descript. des fossiles du groupe nummulitique .... aux environs de Bayonne et de Dax. M. S. G. F., Ser. 2, Bd. 3. 1848.

16. C. Dépérex et F. Roman. Monographie des Pectinidés néogènes de l'Europe et des regions voisines. M. S. G. F. Palaeont. Bd. 10, Abt. 1. No. 26. Teil 1 Genre Pecten.

17. Despayes. Descript. des animaux invertebres dans le bassin de Paris.

18. Alc. D'Orbigny. Voyage dans l'Amérique Méridionale, Bd. 3, Teil 4, Palaeont. Paris 1842.

19. Alc. D'Orbigny, Palaeont. Française, Terrains crétacés Lamellibranches, Paris 1843.

20. M. Douvillé. Essai de classification systémat. des Pectinidés. B. S. G. F. III. Ser., Bd. 25. 1897.

21. Eug. Dumontier. Etud. Palaeont. sur les dépôts jurassiques du bassin du Rhône, Paris 1864-74.

22. P. Fischer. Manuel de Conchyliologie, Paris 1887.

23. Tr. Fucrs. Beitr. z. Kenntnis d. Miocaenfauna Aegyptens a. d. Lybischen Wüste. Palaeontographica, Bd. 30.

24. AUg. Goldfuss. Petrefacta Germaniae, Bonn 1826- 40 . 
25. C. Gortsche. Ueber jurassische Verstein. aus der argent. Cordillere, in "Stelzner: Beitr. z. Geol. u. Palaeont. der argent. Republik." Palaeont. Suppl.3, Lief. 2, 1878.

26. E. Jaworski. Beitr. z. Kenntn. d. Jura in S.-Amerika. Beitr. z. Geol. u. Palaeont. v. S.-Amerika. Herausg. v. G. Steinmann. XXI. N. J. Bl. Bd. 37, 1913.

27. T. Jackson. Phylogeny of the Pelecypoda. The Aviculidae and their allies. Mem. Soc. Nat. Hist. Boston. Bd. 4, No. 8, 1890.

28. MAYer-Eymar. Systemat. Verzeichnis der Kreide- u. Tertiär-Verstein. der Umgegend von Thun. Beitr. z. geol. Karte d. Schweiz. Bd. 24, 2. Beil. 1887.

29. W. Mozricke. Verstein. des Lias u. Unteroolith von Chile. Beitr. zur Geol. u. Palaeont. v. S.-Amerika. Herausg. v. G. Steinmann. N. J. Bl. Bd. No. 9, 1904.

30. H. Nyst. Descript. des coquilles et des polypes fossiles des terrains tert. de la Belgique. Acad. Royal de Bruxelles, Bd. 17. $18+3$.

31. E. PhILIPpr. Beitr. z. Morphologie u. Phylogenie der Lamellibranchier. II. Zur Stammesgeschichte der Pectiniden. Z. D. G. G. Bd. 52, 1900.

32. R. Philippr. Los fosiles secondarios de Chile. Santiago de Chile 1893.

33. AUg. Quenstedt. Der Jura. Tüb. 1858.

34. F. Roemer. Die Verstein. des N. Deutschen Oolithgebirges. Hannover 1836. Suppl. 1839.

35. F. Roemer. Die Kreidebildungen in Texas und ihre organischen Einschlüsse. Bonn 1852.

36. S. Roth, F. Kurtz et C. Burckhardt. Le Lias de la Piedra Pintada (Neuquen). Revista del Museo de la Plata, Bd. 10.

37. F. SAcco. I molluschi del Piemonte et della Liguria, Teil 21-25, Turin 1896-98.

38. T. W. Stanton. The marine cretaceous invertebrates. In „Reports of the Princeton University exped. to Patagonia". 1896-99. Bd. 4. Palaeont. Stuttgart 1901.

39. Otтo Schlagintweit. Die Fauna des Vracon und Cenoman in Perú. In den "Beitr. z. Geol. u. Palaeont. v. S.-Amerika. Heransg. v. G. SteInmanN, XVII, N. J., Bl. Bd. 23.

40. J. Sowerby. The mineral Conchology of Great Britain, Bd. 1-6. London 1812-29.

41. G. Steinmann. Zur Kenntnis der Jura- und Kreideformation von Caracoles (Bolivien). N. J., BI. Bd. 1.

42. A. Thevenne. Palaeont. de Madagaskar. 5. Fossiles liasiques. Ann. de Palaeont. 1908.

43. DE Vernedil et Ed. Collomb. Coup d'oeil sur la constitution géol. de quelques provinces de 1'Espagne. B. S. G.F. Ser. 2, Bd. 10. 1852.

B. S. G. F. = Bulletin société géolog. de France.

M.S. G.F. = Memoires société géolog. de France.

Z. D. G. G. = Zeitschrift d. Dentsch-Geolog. Gesellschaft.

N.J. = Neues Jahrbuch f. Min., Geol. u. Palaeont. 


\section{Inhaltsübersicht}

Einleitung . . . . . . . . . . . . . . . . . 273

1. Teil: Beschreibung des Materials . . . . . . . . . . . . . 274

A. Lias-Volen. . . . . . . . . . . . . . . . . . . . . . 274

a) V. alata (v. Buch) BAYLE et ConU. . . . . . . . . . . . . 277

b) " " " " " $"$ var. Titan \[ör. . . . . . . 283

c) Domeykoi Phil. . . . . . . . . . . . . . . . . . . . . . . . 284

d) Bodenbenderi Benr. . . . . . . . . . . . . . . . . . . 285

B. Pectiniden der Pradoanus-Gruppe. . . . . . . . . . . . 289

a) Pecten Pradoanus Vern. et Coll. . . . . . . . . . . . . . . 289

b) " cordioides PHIL. . . . . . . . . . . . . . . . . . 290

II. Teil: Zur Stammesgeschichte der Gattung Vola. . . . . . . . . . . 291

A. Gliederung der Lias-Volen Südamerikas . . . . . . . . . 291

B. Beziehungen der Lias-Volen Südamerikas zu den jüngeren Volen und Pectiniden . . . . . . . . . . . . . . . . . 292

a) Eozän-Janiren . . . . . . . . . . . . . . . . . . . . 292

b) Oligozän-Janiren . . . . . . . . . . . . . . . . . . . 293

c) Miozäne und postmiozäne Janiren . . . . . . . . . . . . . 294

1. Gruppe II: der Bodenbenderi-Stamm . . . . . . . . . . 294

a) Tertiär-Pectiniden mit Beziehnngen zu den Volen des Bodenbenderi-Stammes . . . . . . . . . . . . . . . . . 296

2. Gruppe I: der Alata-Stamm . . . . . . . . . . . . . 296

a) Abteilung A: Alata-Stamm s. str. . . . . . . . . . . 296

§) " B: Domeykoi-Stamm . . . . . . . . . . . 297

y) Formen des Alata-Stammes s. str. mit flachen, breiten Rippen der Lk. Kl. . . . . . . . . . . . . . . . . . . 298

ס) Formen des Domeykoi-Stammes mit flachen, breiten Rippen der Lk. Kl. . . . . . . . . . . . . . . . . . . 299

ह) Vola-Formen mit reduzierter Berippung der Lk. Kl. . . . . 299

Ђ) Jacobaea- und Aduncus-Gruppe mit sekundär komplizierter Berippung. . . . . . . . . . . . . . . . . . 300

ๆ) Tertiär-Pectiniden mit Beziehungen za den Vola-Formen des Alata-Stammes . . . . . . . . . . . . . . . 301

C. Vola in der Kreide. . . . . . . . . . . . . . . . 304

a) Kreide-Volen mit einfacher gleich starker Berippung der ganzen Klappe 304

b) Kreide-Volen mit ganz geringen Unterschieden in der Stärke der Berippung . . . . . . . . . . . . . . . . . . . . . 306

D. Liasische Pectiniden mit Beziehungen zu Lias-Volen. . . . 306

E. Zusammenfassung . . . . . . . . . . . . . . . . . . . 310

F. Folgerungen . . . . . . . . . . . . . . . . . . . . . 311

G. Literaturverzeichnis . . . . . . . . . . . . . . . 318 